\title{
Proposal for a Nanomechanical Qubit
}

\author{
F. Pistolesi®, ${ }^{1, *}$ A. N. Cleland $\odot,{ }^{2}$ and A. Bachtold $\odot^{3}$ \\ ${ }^{1}$ Université de Bordeaux, CNRS, LOMA, UMR 5798, F-33400 Talence, France \\ ${ }^{2}$ Pritzker School of Molecular Engineering, University of Chicago, Chicago, Illinois 60637, USA \\ ${ }^{3}$ ICFO-Institut de Ciencies Fotoniques, The Barcelona Institute of Science and Technology, \\ 08860 Castelldefels, Barcelona, Spain
}

(Received 31 August 2020; revised 5 May 2021; accepted 24 June 2021; published 3 August 2021)

\begin{abstract}
Mechanical oscillators have been demonstrated with very high quality factors over a wide range of frequencies. They also couple to a wide variety of fields and forces, making them ideal as sensors. The realization of a mechanically based quantum bit could therefore provide an important new platform for quantum computation and sensing. Here, we show that by coupling one of the flexural modes of a suspended carbon nanotube to the charge states of a double quantum dot defined in the nanotube, it is possible to induce sufficient anharmonicity in the mechanical oscillator so that the coupled system can be used as a mechanical quantum bit. However, these results can only be achieved when the device enters the ultrastrong coupling regime. We discuss the conditions for the anharmonicity to appear, and we show that the Hamiltonian can be mapped onto an anharmonic oscillator, allowing us to work out the energy level structure and find how decoherence from the quantum dot and the mechanical oscillator is inherited by the qubit. Remarkably, the dephasing due to the quantum dot is expected to be reduced by several orders of magnitude in the coupled system. We outline qubit control, readout protocols, the realization of a CNOT gate by coupling two qubits to a microwave cavity, and finally how the qubit can be used as a static-force quantum sensor.
\end{abstract}

DOI: 10.1103/PhysRevX.11.031027

Subject Areas: Condensed Matter Physics

\section{INTRODUCTION}

Mechanical systems have important applications in quantum information and quantum sensing-with, for example, significant recent interest in their use for frequency conversion between optical and microwave signals [1-6], the sensing of weak forces using position detection at or beyond the standard quantum limit [7], and demonstrations of mechanically based quantum buses and memory elements [8-12]. Realizing a quantum bit (qubit) based on a mechanical oscillator is thus a highly desirable goal, providing the quantum information community with a new platform for quantum information processing and storage with a number of unique features. A hallmark of mechanical resonators is their ability to couple to a variety of external perturbations, as any force leads to a mechanical displacement; a mechanical qubit could thus enable quantum sensing [13] of a wide range of force-generating

\footnotetext{
*Corresponding author. Fabio.Pistolesi@u-bordeaux.fr

Published by the American Physical Society under the terms of the Creative Commons Attribution 4.0 International license. Further distribution of this work must maintain attribution to the author(s) and the published article's title, journal citation, and DOI.
}

fields. Another outstanding aspect is that mechanical oscillators can be designed to exhibit very large quality factors $[14,15]$, thus well isolated from their environment, with correspondingly long coherence times. Mechanical devices may offer the possibility to develop quantum circuits with both a large number of qubits and a long qubit decoherence time. This possibility is of considerable relevance to quantum computing since the decoherence times of superconducting qubits integrated in large-scale circuits [16] are reduced to of order $10 \mu \mathrm{s}$, which is much lower than what can be achieved when operating single superconducting qubits [17].

A mechanical oscillator can be made into a qubit by introducing a controlled anharmonicity, thereby introducing energy-dependent spacing in the oscillator's quantized energy spectrum $[18,19]$. The anharmonicity then enables the controlled and selective excitation of energy states of the system, for example, the ground and first excited state, without populating other states, breaking the strong correspondence principle that otherwise limits the quantum control of harmonic systems.

Notwithstanding the apparent simplicity of this idea, finding mechanical oscillators with sufficiently strong and controllable anharmonicity is not trivial. In Refs. [18,19], anharmonicity induced by proximity to a buckling instability has been proposed. However, such a scheme is 
(a)

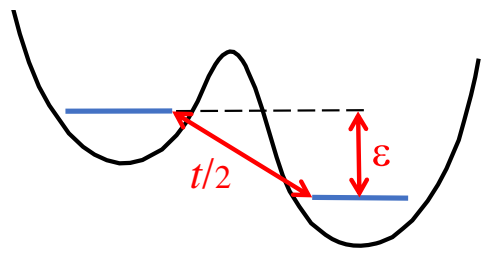

(b) Double-quantum dot

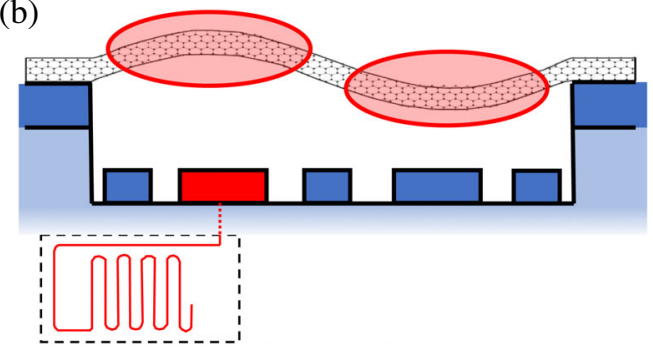

FIG. 1. Schematic of the proposed setup. A suspended carbon nanotube hosting a double quantum dot, whose one-electron charged state is coupled to the second flexural mode. (a) Sketch of the electronic confinement potential and of the two main parameters, the hopping amplitude $t$ and the energy difference $\epsilon$ between the two single-charge states. (b) Physical realization. One of the gate electrodes is connected to a microwave cavity for dispersive qubit readout.

difficult to achieve experimentally. Here, we consider the possibility of coupling one of the flexural modes of a carbon nanotube to an integrated double quantum dot, with the dot itself defined in the nanotube (cf. Fig. 1). By independently tuning the gate voltages for the two quantum dots, it is possible to select the low-energy electronic states so that only those with a single (additional) electron on the double quantum dot are energetically accessible. The excess electron can sit either on the left or the right dot. This charged two-level system is electrostatically coupled to the displacement of the oscillator, in particular, to the second flexural mode, as illustrated in Fig. 1.

In the following, we show that for sufficiently strong electromechanical coupling, the double quantum dot induces a bistability in the mechanical mode by reducing and then changing the sign of the quadratic term of the effective mechanical potential. We find that for strong, but nonetheless reachable coupling constants, it is possible, in this way, to generate an anharmonicity that is sufficient to transform the mechanical oscillator into a qubit; however, this process requires entering the so-called ultrastrong coupling regime, where the coupling strength is larger than the mechanical energy level spacing.

Remarkably, we also find that in the dispersive limit of large detuning of the oscillator frequency and the electronic two-level system energy splitting, the problem can be mapped onto the Hamiltonian of the quantum-anharmonic oscillator, allowing us to use results from that system in this work. Following a description of the anharmonically coupled system, we investigate the decoherence induced by the charged two-level system on the mechanical qubit, as well as how standard protocols for quantum manipulation can be implemented. The reduction of the puredephasing rate of the mechanical qubit with respect to that of the charged two-level system can be made larger than $10^{3}$ with parameters accessible experimentally. We show how qubit readout and manipulation can be achieved as well as how a CNOT gate for two nanomechanical qubits could be realized by coupling them to the same microwave cavity. We also show that the mechanical qubit can be used as a quantum sensor for any static force that could displace the oscillator. The static-force sensitivity can reach values as $\operatorname{good}$ as $10^{-21} \mathrm{~N} / \mathrm{Hz}^{1 / 2}$.

\section{MODEL}

We consider a nanomechanical system [20-27] based on a suspended carbon nanotube (cf. Fig. 1) similar to those demonstrated by a number of groups [28-31]. It has been shown that it is possible to use multiples gates to fine-tune the electrostatic potential along the suspended part of the nanotube $[29,32,33]$. It is thus possible to form a double-well potential to engineer a double quantum dot. We consider the case when only two states, each with one excess electron, are energetically accessible [34], the other states being at higher energy due to the Coulomb interaction. The two single-charge states, corresponding to an electron on the left or right dot, are coupled by a hopping term $t / 2$. Their relative energy difference $\epsilon$ can be controlled by varying the two gate voltages. The two states couple to the nanotube flexural modes. By placing the double dot in the center of the nanotube, the coupling of the two charge states with the second (antisymmetric) mechanical mode is maximized (cf. Fig. 1).

A model Hamiltonian capturing the basic physics of this system can be written as

$$
H=\frac{p^{2}}{2 m}+\frac{m \omega_{\mathrm{m}}^{2} x^{2}}{2}+\frac{\epsilon}{2} \sigma_{z}+\frac{t}{2} \sigma_{x}-\hbar g \frac{x}{x_{\mathrm{z}}} \sigma_{z},
$$

where the first two terms describe the relevant mechanical mode of frequency $\omega_{\mathrm{m}} / 2 \pi$ with effective mass $m$, displacement $x$, and momentum $p$, and we have introduced the zero-point quantum fluctuation $x_{\mathrm{z}}=\left(\hbar / 2 m \omega_{\mathrm{m}}\right)^{1 / 2}$, with $\hbar$ the reduced Planck constant. The electronic response has been reduced to a two-level system, where the two Pauli matrices $\sigma_{z}$ and $\sigma_{x}$ represent the dot charge energy splitting and interdot charge hopping, respectively. Finally, $\hbar g / x_{\mathrm{z}}$ is the variation of the force acting on the mechanical mode when the charge switches from one dot to the other. The value and sign of $g$ can be tuned over a large range by adjusting the gate voltages [14]. In Appendix A, we give a microscopic derivation of the Hamiltonian with the explicit form of the coupling terms. 


\section{BORN-OPPENHEIMER PICTURE}

To gain insight into the physics of the problem, it is instructive to first consider a semiclassical BornOppenheimer picture valid for $\hbar \omega_{\mathrm{m}} \ll \sqrt{t^{2}+\epsilon^{2}}$. We diagonalize $H$ given by Eq. (1), neglecting the $p^{2}$ term and regarding $x$ as a classical variable. The two eigenvalues read

$$
\varepsilon_{ \pm}(x)=m \omega_{\mathrm{m}}^{2} x^{2} / 2 \pm \sqrt{\left(\epsilon-2 \hbar g x / x_{\mathrm{z}}\right)^{2}+t^{2}} / 2 .
$$

In the spirit of the Born-Oppenheimer approximation, the energy profile $\varepsilon_{ \pm}$can be regarded as an effective potential for the oscillator, which depends on which charge quantum level is occupied. Taylor-expanding $\varepsilon_{ \pm}(x)$ for small $x$ and $\epsilon=0$, one finds

$\varepsilon_{ \pm}= \pm \frac{t}{2}+\frac{m \omega_{\mathrm{m}}^{2}}{2}\left(1 \pm \frac{4 \hbar g^{2}}{\omega_{\mathrm{m}} t}\right) x^{2} \mp \frac{4 m^{2} \omega_{\mathrm{m}}^{2} \hbar^{2} g^{4}}{t^{3}} x^{4}+\ldots$

The coupling to the double dot leads to a renormalization of the quadratic coefficient and the appearance of quartic and higher terms. The interaction stiffens the resonating frequency of the upper branch while softening the lower one. In particular, for $g>g_{c}^{\mathrm{sc}}=\left(\omega_{\mathrm{m}} t / 4 \hbar\right)^{1 / 2}$, the quadratic coefficient of the lower branch becomes negative, which leads to a double-well potential and a bistability similar to that predicted for a single quantum dot coupled to a mechanical oscillator [25,27,35-37].

Figure 2 shows the evolution of the two branches of the potential as a function of the coupling constant $g$ for an experimentally accessible value of $t=20 \hbar \omega_{\mathrm{m}}$. One clearly sees the formation of the double-well potential for $g>g_{c}^{\mathrm{sc}}$. For $g=g_{c}^{\mathrm{sc}}$, the potential of the lower branch is purely

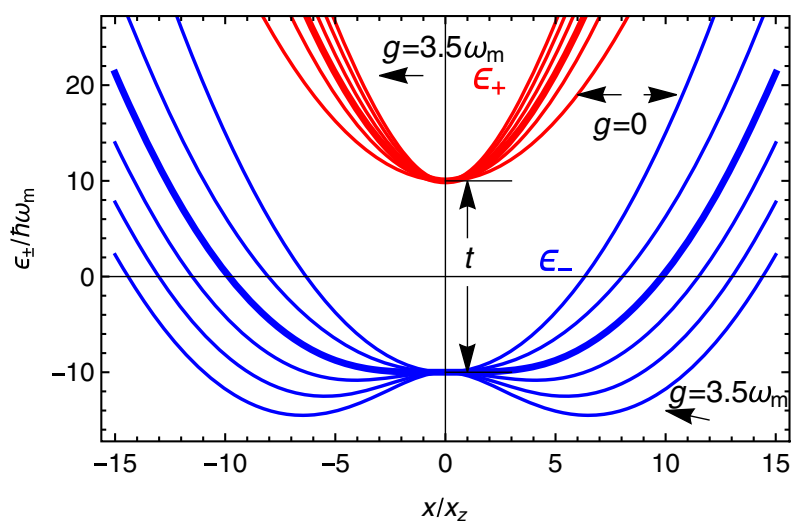

FIG. 2. Effective potentials $\varepsilon_{+}(x)$ (red) and $\varepsilon_{-}(x)$ (blue) from Eq. (2) for $t / \hbar \omega_{\mathrm{m}}=20$ and the values of $\left(4 \mathrm{~g} / \omega_{\mathrm{m}}\right)^{2}=0,10,20$, $30,40,50$, with the first and last lines explicitly indicated in the figure. The potential for $g=g_{c}^{\mathrm{sc}}=\omega_{\mathrm{m}} \sqrt{5}$ is shown with a thicker line. quartic (thick line). Thus, one expects that tuning $g$ close to this critical value, it should be possible to modify, over a large range, the ratio between the quadratic and quartic terms and, consequently, tune the degree of anharmonicity of the system at will.

\section{FULL QUANTUM DESCRIPTION}

\section{A. Conditions for anharmonicity}

The validity of the qualitative description of the previous section can be confirmed in the general case by numerical diagonalization of the Hamiltonian given by Eq. (1) in a truncated Hilbert space. Using a basis comprising the $10^{2}$ lowest harmonic oscillator states largely suffices to reach convergence, and we find the Hamiltonian eigenvectors $|n\rangle$ and eigenstates $E_{n}$ for the problem. The result for the lowest set of energy levels is shown in Fig. 3.

We first notice that for $g \sim g_{\mathrm{c}}^{\mathrm{sc}}$, the ground state crosses the lowest noninteracting electronic level, indicated by the dashed line $-t / 2$, preceding the formation of two bound states in the double well. Note that one expects that this crossing should occur for a coupling larger than $g_{\mathrm{c}}^{\mathrm{sc}}$ since, for this value, the problem reduces to a quartic oscillator, for which the ground state has a positive value [38], similar to the harmonic oscillator zero-point motion $\hbar \omega_{\mathrm{m}} / 2$. For $g \gg g_{\mathrm{c}}^{\mathrm{sc}}$, the above-mentioned bound states have the same energy (cf. the upper-right inset in Fig. 3) and are sufficiently far from each other that their overlap is negligible. In Fig. 3, the third level remains well separated from the first two and merges with the fourth level for large $g$. We introduce the transition frequencies $\omega_{n m}=\left(E_{n}-E_{m}\right) / \hbar$. The anharmonicity, defined as

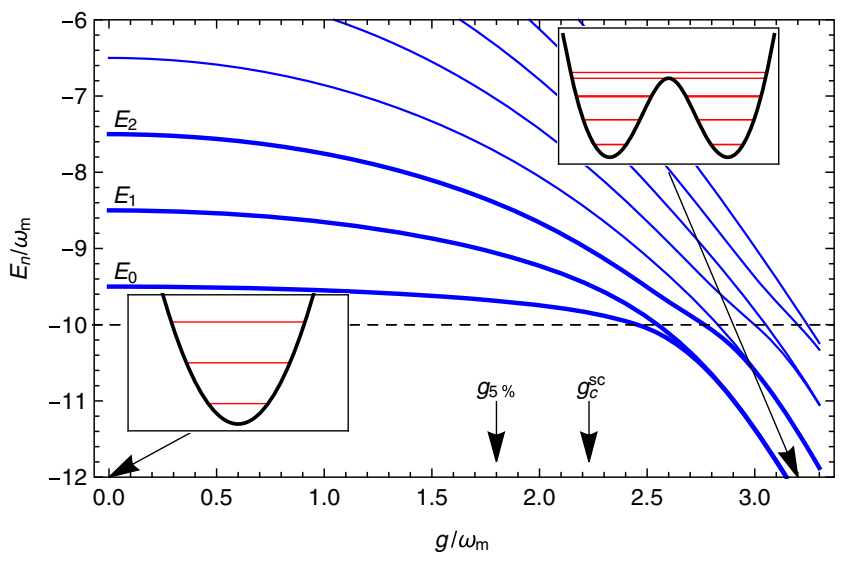

FIG. 3. Lowest-lying energy eigenvalues $E_{n}$ of the Hamiltonian (1) for $\epsilon=0$ and $t=20 \hbar \omega_{\mathrm{m}}$ as a function of $g / \omega_{\mathrm{m}}$. The BornOppenheimer potential given by Eq. (2) and the energy levels are shown in the insets for $g=0$ and $g=3.2 \omega_{\mathrm{m}}$. The dashed line indicates the lowest noninteracting electronic level $-t / 2$. The semiclassical critical value for the bistability is $g_{c}^{\mathrm{sc}} / \omega_{\mathrm{m}}=$ $\sqrt{5} \approx 2.23$. The value of $g=g_{5 \%} \approx 1.8 \omega_{\mathrm{m}}$ for which the anharmonicity is $5 \%$ is also shown. 


$$
a=\frac{\omega_{21}-\omega_{10}}{\omega_{10}}
$$

thus diverges as we increase $g$ from 0 to a value of the order of $g_{\mathrm{c}}^{\mathrm{sc}}$. As discussed in the Introduction, this anharmonicity is crucial for enabling quantum control of the qubit formed by the first two levels, $|0\rangle$ and $|1\rangle$. A minimum requirement is that the transition frequency $\omega_{10}$ between $|0\rangle$ and $|1\rangle$ needs to differ from $\omega_{12}$ between $|1\rangle$ and $|2\rangle$ by much more than the spectral linewidth of the states. As a practical example, in the superconducting transmon qubit [39], an anharmonicity of the order of 5\% suffices to afford full quantum control of the qubit states. In the following, we will thus consider 5\% anharmonicity as a (somewhat arbitrary) requirement, which is sufficient to find the relevant coupling scale required to implement the mechanical qubit.

Resorting again to numerical diagonalization, we present in Fig. 4 a contour plot for the dependence of the anharmonicity on the parameters $t$ and $g$. The thick contour line for $a=0.05$ defines the function $g_{5 \%}$, which gives the required coupling to obtain a 5\% anharmonicity. The region for $t<2 \hbar \omega_{\mathrm{m}}$ presents a more complex structure. A weaker coupling is required to reach the needed anharmonicity. However, in this region, the first two levels inherit the properties of the double quantum dot to a large extent, so we will not discuss this further. Here, we explore the mechanical qubit in the parameter range when $t>2 \hbar \omega_{\mathrm{m}}$, so the nature of the two lowest energy states of the coupled system remains mechanical. A sizable anharmonicity can

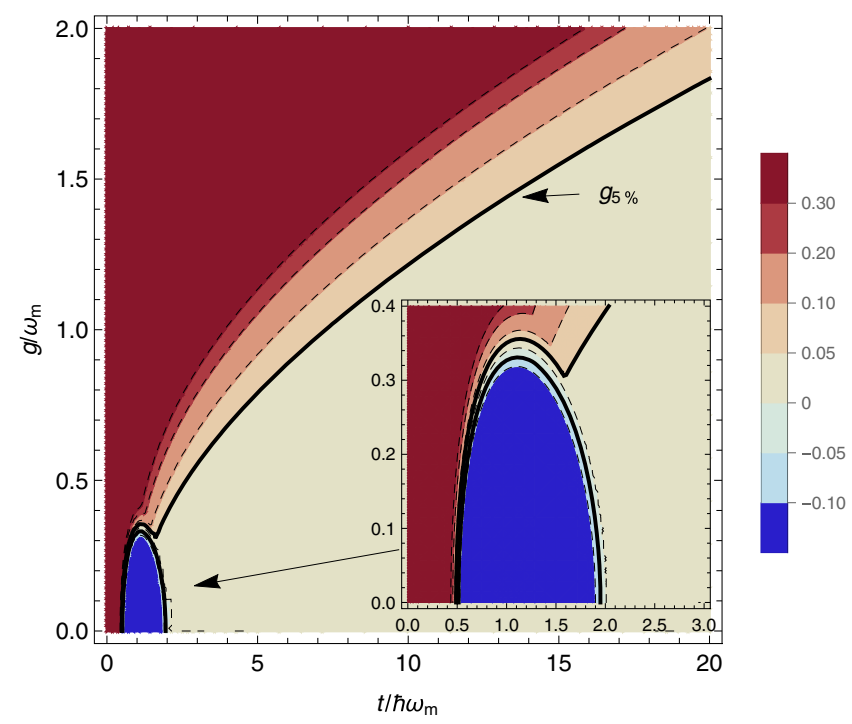

FIG. 4. Contour plot of the anharmonicity $a$ in the $(t, g)$ plane. The contour line for $a=0.05$ is thicker, and it defines the function $g_{5 \%}(t)$. The kink at $t \approx 1.54 \hbar \omega_{\mathrm{m}}$ of this function, better seen in the inset, is due to the avoided crossing between the charge and oscillator eigenstates that occurs at that value of $t$. It indicates the region where the eigenstate begins to have a predominantly charge nature. only be reached when operating the device near or in the ultrastrong coupling regime, $g>\omega_{\mathrm{m}}$, as seen in Fig. 4.

\section{B. Eigenstates}

It is interesting to investigate the nature of the two qubit states $|0\rangle$ and $|1\rangle$. In the position representation, the wave function is given by $\psi_{n \sigma}(x)=\langle x, \sigma \mid n\rangle$, where $|n\rangle$ is the Hamiltonian eigenstate and $|x, \sigma\rangle$ is the eigenstate of the displacement $x$ and $\sigma_{z}$ operators with eigenvalues $x$ and $\sigma$, respectively. The wave function $\psi_{n \sigma}(x)$ can be chosen to be real-valued. Instead of looking directly at $\psi_{n \sigma}(x)$, it is more interesting to consider the averages of the operators $\sigma_{i}$ as a function of $x:\left\langle\sigma_{i}\right\rangle(x)=\sum_{\sigma, \sigma^{\prime}} \psi_{n \sigma}(x)\left[\sigma_{i}\right]_{\sigma \sigma^{\prime}} \psi_{n \sigma^{\prime}}(x)$. Since by symmetry $\left\langle\sigma_{y}\right\rangle=0$, only $\left\langle\sigma_{x}\right\rangle=2 \psi_{n+} \psi_{n-}$ and $\left\langle\sigma_{z}\right\rangle=$ $\psi_{n+}^{2}-\psi_{n-}^{2}$ are nonvanishing.

We display in Fig. 5 these two components as well as the total probability for the oscillator displacement $\psi^{2}=$ $\psi_{n+}^{2}+\psi_{n-}^{2}$ (blue curve in Fig. 5). The function $\left\langle\sigma_{z}\right\rangle(x)$ gives the distribution of the charge (green curve in Fig. 5), while $\left\langle\sigma_{x}\right\rangle(x)$ indicates the strength of the coherent superposition of the two charge states (yellow curve in Fig. 5). These two quantities are in competition. From the figure, one sees that for weak coupling, $\left\langle\sigma_{z}\right\rangle(x) \approx 0$, and the displacement probability distribution coincides with $-\left\langle\sigma_{x}\right\rangle(x)$. At the value of $g=g_{5 \%}$, the distribution of the charge depends on $x$, for both states. Finally, for the bistable case with $g / \omega_{\mathrm{m}}=3.0$, one reaches the limit where $\left|\left\langle\sigma_{z}\right\rangle(x)\right|$ is close to the displacement probability, indicating a full correlation between the displacement and the charge. In the figure, we also show the distribution of the harmonic oscillator states. One clearly sees that for $g=g_{5 \%}$, the two states are still mainly eigenstates of the mechanical oscillator.

\section{Mapping in the dispersive regime}

The numerical diagonalization shows that the semiclassical picture provides a good qualitative description. A natural question is then how far one can extend this picture. For this reason, we look for a unitary transformation $U$ that could map the Hamiltonian given in Eq. (1) onto that of a simple anharmonic oscillator. In the limit of $g /\left|t / \hbar-\omega_{\mathrm{m}}\right| \ll 1$, known as the dispersive limit, we find a $U$ such that, at fourth order in $g$, we can write $H_{T}=U^{\dagger} H U$, with

$H_{T}=\frac{t}{2} \sigma_{z}+\frac{\hbar \omega_{\mathrm{m}}}{4}\left[\alpha_{1} \hat{p}^{2}+\alpha_{2} \hat{x}^{2}+\sigma_{z}\left(\alpha_{3} \hat{x}^{2}+\alpha_{4} \hat{x}^{4}\right)\right]$.

[We discarded the constant $\hbar^{3} g^{2} \omega_{\mathrm{m}} /\left(t^{2}-\hbar^{2} \omega_{\mathrm{m}}^{2}\right)$.] Here, we introduce the quadratures $\hat{x}=x / x_{\mathrm{z}}=a^{\dagger}+a$, $\hat{p}=p /\left(m \omega_{\mathrm{m}} x_{\mathrm{z}}\right)=i\left(a^{\dagger}-a\right)$, with $[\hat{x}, \hat{p}]=2 i$, where $a$ and $a^{\dagger}$ are the creation and destruction operators for the harmonic oscillator eigenstates. The four coefficients read 

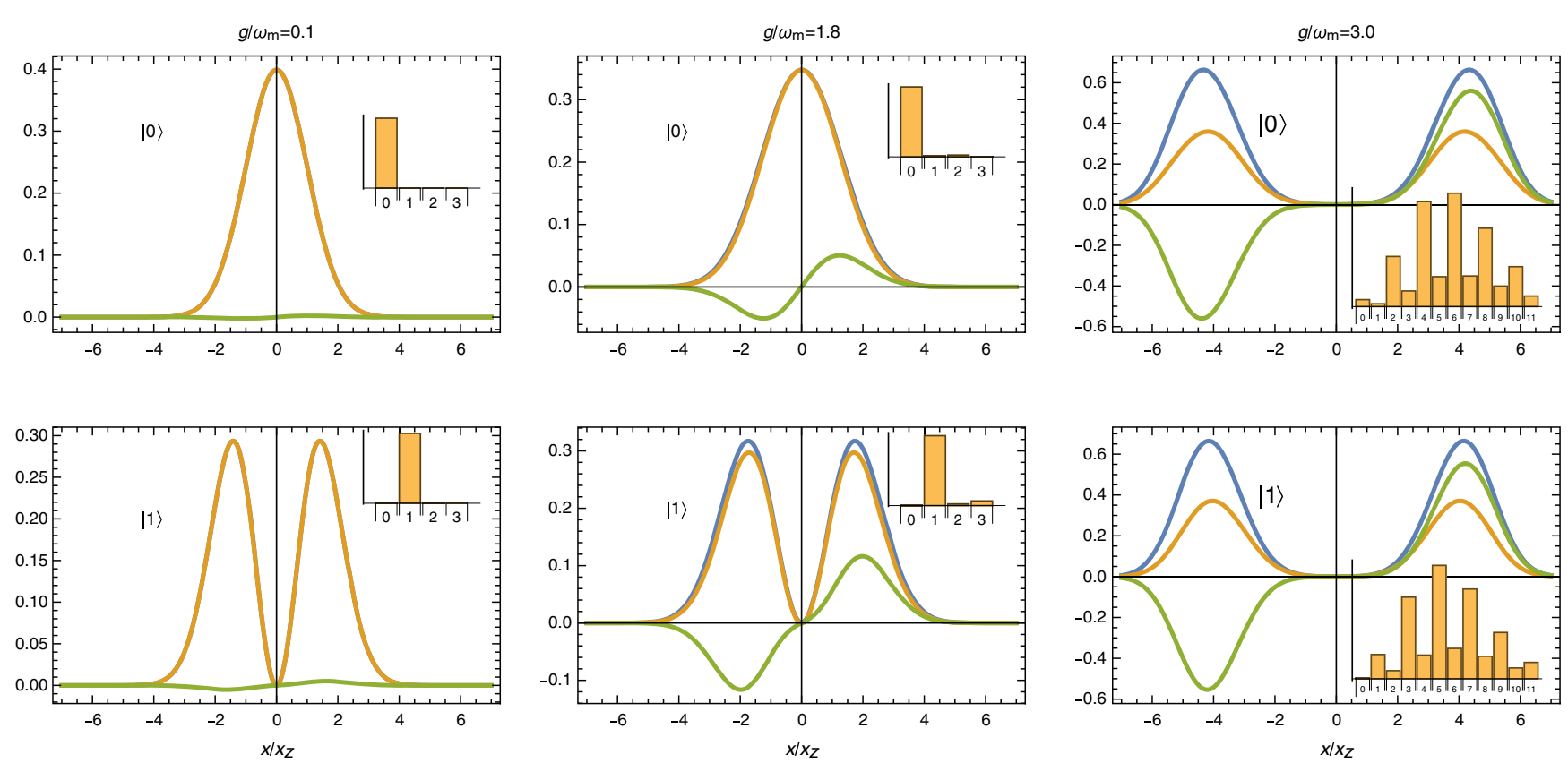

FIG. 5. Wave functions of the two qubit states $|0\rangle$ (upper panels) and $|1\rangle$ (lower panels) for $t / \hbar \omega_{\mathrm{m}}=20, \mathrm{~g} / \omega_{\mathrm{m}}=0.1,1.8$, and 3.0. We plot $-\left\langle\sigma_{x}\right\rangle(x)$ (yellow), $\left\langle\sigma_{z}\right\rangle(x)$ (green), and $\psi_{n+}(x)^{2}+\psi_{n-}(x)^{2}$ (blue). Note that for small coupling, the yellow and blue lines perfectly overlap. The probability of occupation of the first single-harmonic oscillator states is indicated in the insets.

$$
\begin{gathered}
\alpha_{1}=1+\frac{128 \hbar^{6} g^{4} t^{2} \omega_{\mathrm{m}}^{2}}{\Delta^{6} \Delta_{3}^{2}}, \quad \alpha_{2}=1-\frac{16 \hbar^{4} g^{4} t^{2}}{\Delta^{4} \Delta_{3}^{2}}, \\
\alpha_{3}=\frac{4 \hbar t g^{2}}{\omega_{\mathrm{m}} \Delta^{2}}, \quad \alpha_{4}=-\frac{4 \hbar^{3} t g^{4}\left(3 t^{2}+\hbar^{2} \omega_{\mathrm{m}}^{2}\right)}{3 \omega_{\mathrm{m}} \Delta^{6}},
\end{gathered}
$$

where $\Delta^{2}=t^{2}-\left(\hbar \omega_{\mathrm{m}}\right)^{2}, \Delta_{3}^{2}=t^{2}-9\left(\hbar \omega_{\mathrm{m}}\right)^{2}$. The derivation and the definition of $U$ are given in Appendix B.

Remarkably, we find that within this approximation, it is possible to map the problem onto a new description with two anharmonic oscillators, one for each charge branch. The upper branch is unstable if we stop the expansion at $x^{4}$ since it has a negative quartic term. This description thus holds for a small but nonzero value of the ratio $\hbar \omega_{\mathrm{m}} / t$, giving a more accurate description than the simpler BornOppenheimer approach.

The anharmonic oscillator is a well-studied problem [40]. When the quadratic part is positive, it is convenient to write the lower branch of Eq. (5) in the standard form,

$$
H=\hbar \omega_{\mathrm{m}}^{\prime}\left(\hat{x}^{2}+\hat{p}^{2}+\lambda \hat{x}^{4}\right) / 4 \text {. }
$$

This can be done by the scaling $\hat{x}=\xi \hat{x}^{\prime}$ and $\hat{p}=\hat{p}^{\prime} / \xi$, so the commutation relation is preserved $\left[\hat{x}^{\prime}, \hat{p}^{\prime}\right]=2 i$, with

$$
\xi=\left[\alpha_{1} /\left(\alpha_{2}-\alpha_{3}\right)\right]^{1 / 4} .
$$

The renormalized resonant frequency reads $\omega_{\mathrm{m}}^{\prime}=$ $\omega_{\mathrm{m}}\left[\alpha_{1}\left(\alpha_{2}-\alpha_{3}\right)\right]^{1 / 2}$, and the quartic coefficient is

$$
\lambda=\frac{\alpha_{4} \alpha_{1}^{1 / 2}}{\left(\alpha_{2}-\alpha_{3}\right)^{3 / 2}}
$$

Note that we now consider only positive values of $\omega_{\mathrm{m}}^{\prime}$, but Eq. (5) also holds in the bistable region. The anharmonicity $a$ defined in Eq. (4) becomes a function of $\lambda$ only. Using the expression (1.17) of Ref. [40] for the eigenvalues in terms of $\lambda$ and Eq. (10), one can obtain an analytical expression for the anharmonicity in terms of the parameters $\omega_{\mathrm{m}}, t$, and $g$ that agrees with the numerics with reasonable accuracy, as can be seen in Fig. 6. One finds that the 5\% anharmonicity is achieved for $\lambda_{c} \approx 0.0225$ (the exact numerical result is $\lambda_{c}=0.0220$ ).

\section{Operators acting on the qubit}

In order to study the control, readout, and decoherence of the qubit formed by the two states $|0\rangle$ and $|1\rangle$, it is necessary to find the projection of the physical operators $\sigma_{i}, \hat{x}$, and $\hat{p}$ in the Hilbert space spanned by $\{|0\rangle,|1\rangle\}$. In this space, any operator can be written as a linear combination of the unit matrix $\left(\tau_{0}\right)$ and the three Pauli matrices, which we define here as $\left\{\tau_{x}, \tau_{y}, \tau_{z}\right\}$, to distinguish them from the operators $\sigma_{i}$ acting in the charge space. The Hamiltonian of the qubit then simply reads $\hbar \omega_{10} \tau_{z} / 2$. One can calculate numerically the matrix elements of any operator in the qubit subspace and then obtain its form in terms of a sum of the four $\tau$ matrices. We find, for the charge variables [in the representation of Eq. (1)], 


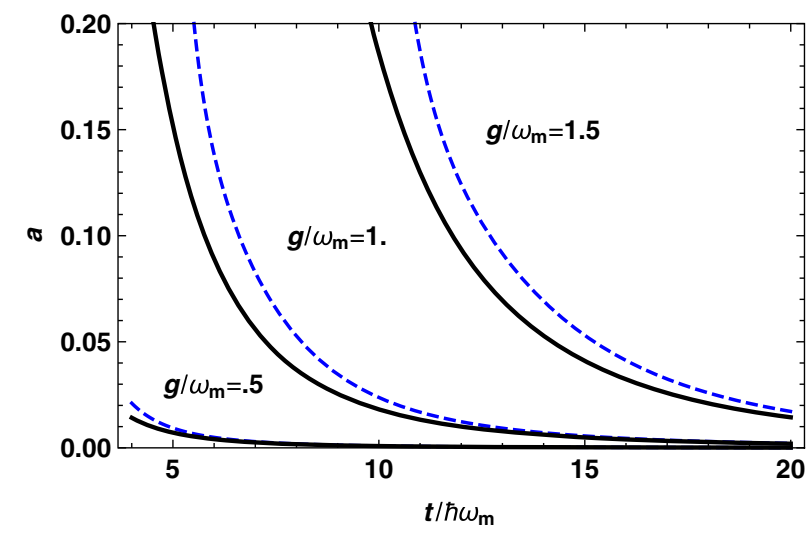

FIG. 6. Comparison between numerical (solid line) and analytical (dashed line) dependence of the anharmonicity parameter $a$ for three values of the coupling $g / \omega_{\mathrm{m}}=0.5,1$, and 1.5 .

$$
\begin{aligned}
\left.\sigma_{x}\right|_{\mathrm{qb}} & =\beta_{1} \tau_{0}+\beta_{2} \tau_{z} \\
\left.\sigma_{y}\right|_{\mathrm{qb}} & =\beta_{3} \tau_{y} \\
\left.\sigma_{z}\right|_{\mathrm{qb}} & =\beta_{4} \tau_{x},
\end{aligned}
$$

and for the oscillator variables

$$
\begin{aligned}
& \left.\hat{x}\right|_{\mathrm{qb}}=\beta_{5} \tau_{x} \\
& \left.\hat{p}\right|_{\mathrm{qb}}=\beta_{6} \tau_{y} .
\end{aligned}
$$

The six coefficients can be obtained numerically, but it is also interesting to obtain approximate analytical expressions for them, which can be achieved by using the unitary transformation introduced above (see Appendix B):

$$
\begin{gathered}
\beta_{1}=-1+4(\hbar g)^{2} \frac{\left(\hbar \omega_{\mathrm{m}}\right)^{2}-t \hbar \omega_{\mathrm{m}} \xi^{2}+t^{2} \xi^{4}}{\Delta^{4} \xi^{2}}+g^{4} \beta_{1,4}, \\
\beta_{2}=-2(\hbar g)^{2} \frac{\left(\hbar \omega_{\mathrm{m}}\right)^{2}+t^{2} \xi^{4}}{\Delta^{4} \xi^{2}}+g^{4} \beta_{2,4}, \\
\beta_{3}=\frac{2 \hbar^{2} g \omega_{\mathrm{m}}}{\Delta^{2} \xi}+g^{3} \beta_{3,3}, \\
\beta_{4}=\frac{2 \hbar g t \xi}{\Delta^{2}}+g^{3} \beta_{4,3}, \\
\beta_{5}=\xi-\frac{2 \hbar^{3} g^{2} t \omega_{\mathrm{m}} \xi}{\Delta^{4}}+g^{4} \beta_{5,4}, \\
\beta_{6}=\frac{1}{\xi}-\frac{2 \hbar^{3} g^{2} t \omega_{\mathrm{m}}}{\Delta^{2} \xi}+g^{4} \beta_{6,4} .
\end{gathered}
$$

The coefficients for $g^{3}$ and $g^{4}$ are given by Eqs. (B15)(B20) in Appendix B.

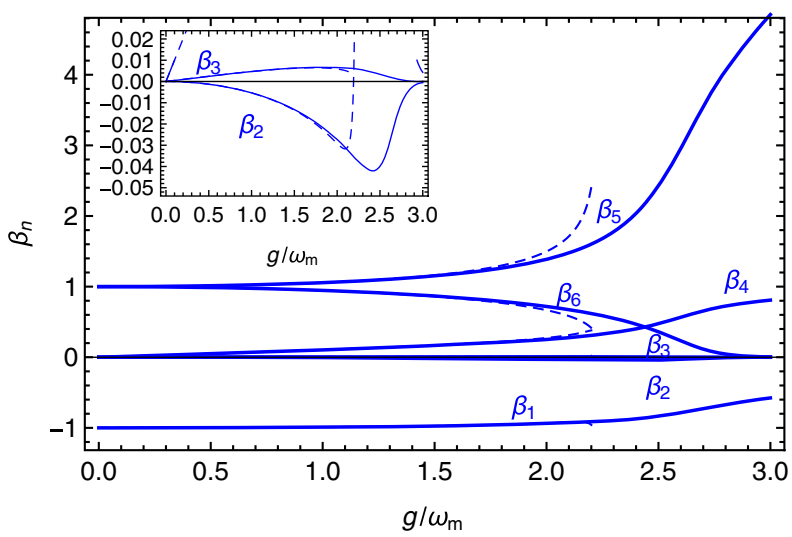

FIG. 7. Coefficients $\beta_{i}$ of the operator projections in the qubit space, obtained by numerical diagonalization (solid lines) and from the analytical approximation to fourth order in $g$ (dashed lines). The value of $t$ is fixed here to $20 \hbar \omega_{\mathrm{m}}$.

We show in Fig. 7 the behavior of the analytic coefficients as a function of $\mathrm{g} / \omega_{\mathrm{m}}$ for $t / \hbar \omega_{\mathrm{m}}=20$ and compare to the exact numerical results. The analytical expressions again give a good description in the interesting range $g<g_{5 \%}$. In particular, these expressions allow us to recognize that $\beta_{2}$ and $\beta_{3}$ are parametrically small for $g \approx \omega_{\mathrm{m}} \ll t / \hbar$.

Another important result given by the expressions for the $\beta_{i}$ is the charge component of the qubit. This component can be identified with the value of the $\beta_{4}$ coefficient, which gives the projection of the charge operator $\sigma_{z}$ in the qubit space. This coefficient vanishes linearly in $g$, and it remains small up to $g \approx \sqrt{\omega_{\mathrm{m}} t / \hbar}$ when $t \gg \hbar \omega_{\mathrm{m}}$. In this case, we thus expect that the qubit has a predominantly mechanical character in its degrees of freedom, measured by the $\beta_{5}$ and $\beta_{6}$ coefficients, which remain of the order of unity.

\section{E. Qubit manipulation}

The values of $\beta$ are also crucial to understanding how to manipulate the qubit. This is achieved using a completely classical oscillating voltage applied to a nearby wire, turned on for some duration with a calibrated amplitude. The anharmonicity of the system allows this classical signal to achieve quantum control. One can find the effect of an oscillating voltage on the qubit by considering how this voltage couples to the $\sigma_{i}$ and $\hat{x}$ operators. In Appendix A, we derive these couplings for a potential $V_{g 12}^{\text {ac }}$ applied to the two gates controlling the electrochemical potential of each dot [cf. Eq. (A24)]. We find that the potential couples to $\sigma_{z}$ and $\hat{x}$ with the coefficients $\lambda^{\mathrm{ev}}$ and $\lambda^{\mathrm{mv}} x_{\mathrm{z}}$, respectively (see Appendix A for the explicit expressions). Since both $\hat{x}$ and $\sigma_{z}$ project onto $\tau_{x}$, we find that the coupling to the oscillating field is just $\lambda_{v} \tau_{x} V_{g 12}^{\text {ac }}$, with

$$
\lambda_{v}=\lambda^{\mathrm{mv}} x_{\mathrm{z}} \beta_{5}+\lambda^{\mathrm{ev}} \beta_{4}
$$


This result indicates that one can use standard methods to manipulate the qubit state, e.g., by using nuclear magnetic resonance methods by driving the qubit states at a frequency $\omega_{D}$ with pulses that induce, in the rotating frame, a term $\hbar\left(\omega_{10}-\omega_{D}\right) \tau_{z} / 2+\lambda_{v} V_{g 12}^{0} \tau_{x}$ [41]. The anharmonicity guarantees that the second excited state will not be populated by these manipulations.

\section{F. Qubit readout}

Reading out the state of the qubit can be realized by coupling the system to a microwave superconducting cavity and using a dispersive interaction, analogous to what is done with superconducting qubits $[42,43]$. The coupling can be obtained from the expression of the coupling to an oscillating voltage [cf. Eqs. (A22) and (A23)] with the substitution $V^{\text {ac }} \rightarrow V_{\mathrm{z}}\left(b+b^{\dagger}\right)$, where $b$ is the destruction operator of the photons in the cavity and $V_{\mathrm{z}}$ is the zeropoint voltage of the cavity. The coupling Hamiltonian reads

$$
H^{\mathrm{qb}-\mathrm{cav}}=\hbar g_{v} \tau_{x}\left(b+b^{\dagger}\right),
$$

with $\hbar g_{v}=\lambda_{v} V_{\mathrm{z}}$ [cf. Eq. (19)]. A standard method is then to perform a dispersive measurement of the superconducting cavity frequency, modified by an amount that depends on the qubit state. By performing a unitary transformation [44], one can eliminate the term $\tau_{x}$ from the Hamiltonian and obtain, for the qubit and cavity Hamiltonian,

$$
H / \hbar=\omega_{10} \tau_{z} / 2+\left(\omega_{c}+\chi \tau_{z}\right) b^{\dagger} b
$$

where $\omega_{c}$ is the cavity resonant frequency and $\chi=$ $g_{v}^{2} /\left(\omega_{10}-\omega_{c}\right)$ the dispersive frequency shift. Since the resonating frequency now depends on the qubit state, this allows us to perform an efficient, quantum, nondestructive readout of the qubit state.

This picture remains qualitatively correct, but in analogy with what happens in the transmon qubit [45], when the anharmonicity is small, one needs to include the other system states to calculate the dispersive coupling correctly. In Appendix $\mathrm{C}$, we present the calculation of $\chi$ for the problem at hand by using second-order perturbation theory in the coupling constant to the cavity. In this picture, the eigenstates can be labeled according to the branch $(\sigma= \pm)$ in Fig. 2 with $|n \sigma\rangle$ and eigenstate energy $E_{n \sigma}$. We find that the second excited state, $|2\rangle=|2-\rangle$, and two other excited states of the upper branch $(|0+\rangle$ and $|1+\rangle)$ with an excitation energy of the order of $t$ contribute. The parameter $\chi$ entering Eq. (21) reads $\chi=\chi_{m}+\chi_{e}$, with

$$
\chi_{m}\left(\omega_{c}\right) \approx \frac{\left(g_{\mathrm{ec}} \beta_{4,1}\right)^{2}\left(\omega_{21}-\omega_{10}\right)}{\left(\omega_{c}-\omega_{21}\right)\left(\omega_{c}-\omega_{10}\right)}
$$

dominant for $\omega_{c} \approx \omega_{10}$ and

$$
\chi_{e}\left(\omega_{c}\right) \approx \frac{g_{\mathrm{ec}}^{2}\left(\delta_{11}-\delta_{00}\right)}{2\left(\omega_{c}-\delta_{11}\right)\left(\omega_{c}-\delta_{00}\right)},
$$

for $\omega_{c} \approx \delta_{00}$. Here, $\beta_{4,1}=2 \hbar g \xi t / \Delta^{2} \ll 1$ is the first-order contribution to $\beta_{4}$ [cf. Eq. (16)], $\delta_{n m}=\left(E_{n+}-E_{m-}\right) / \hbar$, and $\hbar g_{\mathrm{ec}}=\lambda^{\mathrm{ev}} V_{z}$. One can see that $\chi_{m}$ is proportional to the anharmonicity and thus vanishes in the harmonic case. The expression for $\chi_{e}$ also vanishes when the coupling constant vanishes, but it does not require an anharmonicity:

$$
\delta_{11}-\delta_{00} \approx \frac{4 g^{2} t}{\Delta^{2}}
$$

At lowest order, this value is just the difference of the semiclassical resonating frequencies of the upper and lower branches. This dispersive coupling relies on the intrinsic anharmonicity of the charge two-level system.

We can further simplify Eq. (22) by considering $\omega_{c}$ close to $\omega_{10}$ : The small numerator is compensated by a vanishing denominator, and one obtains $\chi_{m} \approx\left(g_{\mathrm{ec}} \beta_{4,1}\right)^{2} /$ $\left(\omega_{10}-\omega_{c}\right)$, which remarkably coincides with the standard form of the dispersive coupling. Even if this seems independent of the anharmonicity, note that it is necessary that $\left|\omega_{21}-\omega_{10}\right|>g_{\mathrm{ec}} \beta_{4,1}$ for the calculation to be valid; this condition sets the constraint on the anharmonicity $a>g_{\mathrm{ec}} \beta_{4,1} / \omega_{10}$. Choosing the detuning to the minimum value allowed by second-order perturbation theory, $g_{\mathrm{ec}} \beta_{4,1}$, one obtains $\chi_{m}^{\max } \approx g_{\mathrm{ec}} \beta_{4,1}<a \omega_{10}$. Since $\omega_{c} \approx \omega_{10}$, a quality factor larger than $1 / a$ would be largely sufficient to detect the qubit state.

Similar arguments can be applied to the expression for $\chi_{e}$, leading to $\chi_{e}^{\max } \approx g_{\mathrm{ec}}$. In this case, the limitation is less severe since the condition $\left|\delta_{11}-\delta_{00}\right|>g_{\mathrm{ec}}$ does not involve the anharmonicity. Using Eq. (24), we get approximately $g_{\mathrm{ec}}<4 g^{2} / t$. This result suggests that it may be more convenient to tune the cavity to this resonance and exploit the $\chi_{e}$ dispersive coupling to read out the qubit state.
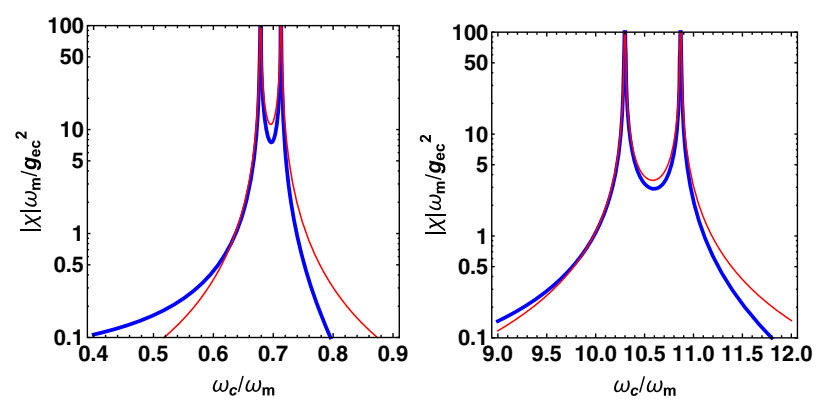

FIG. 8. Quantity $|\chi| \omega_{\mathrm{m}} / g_{\mathrm{ec}}^{2}$ for $t / \hbar \omega_{\mathrm{m}}=10, g / \omega_{\mathrm{m}}=1.2164$ (for which $a=0.05$ ) as a function of $\omega_{c} / \omega_{\mathrm{m}}$ in two different regions of the spectrum: close to $\omega_{10}<\omega_{12}$ and close to $\delta_{00}<\delta_{11}$, left and right panels, respectively. The thick blue line gives the numerical calculation, the thin red line the expressions (22) and (23), shown in the left and right panels, respectively. 
These analytical expressions are obtained as a perturbative expansion in $g / t$, but the expressions remain accurate in the range of coupling of interest for our purposes, as shown as an example in Fig. 8.

\section{DECOHERENCE}

The double quantum dot and the mechanical oscillator are unavoidably coupled to the environment, which induces decoherence and incoherent transitions between energy levels. The decoherence rate of the double quantum dot charge qubit is much larger than that of the mechanical resonator, so it will limit the performances of the mechanical qubit. The best values for the decoherence rate are in the $\mathrm{MHz}$ range [46].

In order to study how the nanomechanical qubit inherits the decoherence of its two subsystem components, we begin by constructing a simple model for the coupling of the subsystems to the environment.

We write the coupling Hamiltonian as

$$
H_{I}=\hat{A}^{\mathrm{c}} \hat{E}_{1}+\hat{x} \hat{E}_{2},
$$

where $\hat{A}^{\mathrm{c}}=\sum_{i=x, y, z} v_{i} \sigma_{i}=\vec{v} \cdot \vec{\sigma}$ is the most general operator in the charge subspace (see, for instance, Ref. [47]). The operators $\hat{E}_{1}$ and $\hat{E}_{2}$ are given by the sum of operators, which, themselves, involve many degrees of freedom that model the environment of the charge and the mechanical oscillator, respectively (the coupling constant is absorbed in the $\hat{E}$ operators so that $\hat{A}^{\mathrm{c}}$ and $\hat{x}$ are dimensionless). We assume that we know the correlation functions $C_{i}(t)=\left\langle\hat{E}_{i}(t) \hat{E}_{i}(0)\right\rangle$, as well as their Fourier transforms $S_{i}(\omega)=\int d t e^{i \omega t} C_{i}(t)$, and that the charge and mechanical environments are independent, $\left\langle\hat{E}_{1}(t) \hat{E}_{2}(0)\right\rangle=0$. If $S_{i}(\omega)$ is a sufficiently smooth function for $\omega$ close to the qubit resonant frequency, the three parameters $v_{i}$ give a complete description of the coupling to the environment of the charge system. For the mechanical oscillator, we parametrize the coupling to the environment with a single damping rate $\gamma$.

One can then use the standard procedure, integrating out the environmental degrees of freedom and finding an equation for the reduced density matrix $\rho$ in the BornMarkov and rotating-wave approximations. The rate equations have the standard form

$$
\begin{gathered}
\dot{\rho}_{n n}=-\rho_{n n} \sum_{p \neq n} \Gamma_{n \rightarrow p}+\sum_{p \neq n} \rho_{p p} \Gamma_{p \rightarrow n}, \\
\dot{\rho}_{n m}=-\left[\sum_{p \neq n} \Gamma_{n \rightarrow p} / 2+\sum_{p \neq m} \Gamma_{m \rightarrow p} / 2+\Gamma_{n m}^{\phi}\right] \rho_{n m},
\end{gathered}
$$

where $\rho_{n m}=\langle n|\rho| m\rangle$ is the matrix element of $\rho$ in the eigenstate basis $|n\rangle$ of the Hamiltonian (1) with eigenvalues $E_{n}$. The rates read

$$
\begin{aligned}
\Gamma_{n \rightarrow m} & =2 \pi S_{1}\left(\omega_{n m}\right)\left|A_{n m}^{\mathrm{c}}\right|^{2}+2 \pi S_{2}\left(\omega_{n m}\right)\left|x_{n m}\right|^{2}, \\
\Gamma_{n m}^{\phi} & =\pi S_{1}(0)\left(A_{n n}^{\mathrm{c}}-A_{m m}^{\mathrm{c}}\right)^{2}+\pi S_{2}(0)\left(x_{n n}-x_{m m}\right)^{2},
\end{aligned}
$$

where $O_{n m}=\langle n|O| m\rangle$ and $\Gamma_{n m}^{\phi}$ is the pure dephasing rate. These equations hold at nonzero temperature $T$, with $S_{i}(\omega)=S_{i}(-\omega) e^{\hbar \omega / k_{B} T}$ where $k_{B}$ is the Boltzmann constant. When only two levels are present, one finds

$$
\begin{gathered}
\dot{\rho}_{00}=-\rho_{00} \Gamma_{0 \rightarrow 1}+\rho_{11} \Gamma_{1 \rightarrow 0}, \\
\dot{\rho}_{01}=-\rho_{01}\left(\Gamma_{0 \rightarrow 1}+\Gamma_{1 \rightarrow 0}+2 \Gamma_{01}^{\phi}\right) / 2 .
\end{gathered}
$$

The last equation defines the coherence time of the qubit $T_{2}=2 /\left(\Gamma_{0 \rightarrow 1}+\Gamma_{1 \rightarrow 0}+2 \Gamma_{01}^{\phi}\right)$. In the following, we focus on the two rates $\Gamma_{1 \rightarrow 0}$ and $\Gamma_{01}^{\phi}$. (We do not consider the case of equally spaced levels inducing transfer of coherence between higher energy states [48].)

\section{A. Noninteracting case}

Let us begin with the noninteracting case $(g=0)$ in order to define the rates. We have two independent systems: the double quantum dot and the mechanical oscillator. For the oscillator, one finds $\Gamma_{1 \rightarrow 0}^{\mathrm{m}}=2 \pi S_{2}\left(\omega_{\mathrm{m}}\right)=$ $\gamma\left(1+n_{\mathrm{th}}\right)$, where $n_{\mathrm{th}}=1 /\left(e^{\hbar \omega_{\mathrm{m}} / k_{B} T}-1\right)$ and $\Gamma_{12}^{\mathrm{m}, \phi}=0$. For the charge system, we begin by diagonalizing the Hamiltonian $H_{0}=\left(\epsilon \sigma_{z}+t \sigma_{x}\right) / 2$, performing a rotation by an angle $\theta=\arctan (t / \epsilon)$ around the $y$ axis: $U(\theta)=e^{-i \theta \sigma_{y} / 2}$. One has

$$
\begin{aligned}
& U(\theta)^{\dagger} \sigma_{x} U(\theta)=\cos \theta \sigma_{x}-\sin \theta \sigma_{z}, \\
& U(\theta)^{\dagger} \sigma_{z} U(\theta)=\sin \theta \sigma_{x}+\cos \theta \sigma_{z},
\end{aligned}
$$

with $\sigma_{y}$ invariant. The charge Hamiltonian coupled to the environment then becomes

$$
H^{\prime}=U^{\dagger} H U=\frac{1}{2} \sqrt{t^{2}+\epsilon^{2}} \sigma_{z}+\overrightarrow{v^{\prime}} \vec{\sigma} \hat{E}_{1},
$$

with $v_{x}^{\prime}=\cos \theta v_{x}+\sin \theta v_{z}, v_{z}^{\prime}=-\sin \theta v_{x}+\cos \theta v_{z}$, and $v_{y}^{\prime}=v_{y}$, which gives the rates

$$
\begin{gathered}
\Gamma_{1 \rightarrow 0}^{\mathrm{c}}(\theta)=2 \pi S_{1}\left(\sqrt{\epsilon^{2}+t^{2}}\right)\left[\left(\cos \theta v_{x}+\sin \theta v_{z}\right)^{2}+v_{y}{ }^{2}\right] \\
\Gamma_{01}^{\mathrm{c}, \phi}(\theta)=4 \pi S_{1}(0)\left(\sin \theta v_{x}-\cos \theta v_{z}\right)^{2}
\end{gathered}
$$

According to these equations, the pure dephasing and decay rates depend on the value of $\theta$ (i.e., the ratio $\epsilon / t$ ). Since the environmental spectrum depends only on the charge energy splitting, the ratios 


$$
\begin{gathered}
R^{D} \equiv \frac{\Gamma_{0 \rightarrow 1}^{\mathrm{c}}(0)}{\Gamma_{0 \rightarrow 1}^{\mathrm{c}}(\pi / 2)}=\frac{v_{x}^{2}+v_{y}^{2}}{v_{z}^{2}+v_{x}^{2}}, \\
R^{\phi} \equiv \frac{\Gamma_{01}^{\mathrm{c}, \phi}(0)}{\Gamma_{01}^{\mathrm{c}, \phi}(\pi / 2)}=\frac{v_{z}^{2}}{v_{x}^{2}}
\end{gathered}
$$

depend only on the values of $v_{i}$. One can then, at least in principle, measure the rates for the same energy splitting $\sqrt{t^{2}+\epsilon^{2}}$ and the two values of $\theta, 0$ and $\pi / 2$. This approach gives $R^{D}$ and $R^{\phi}$, which can be used to express $v_{y}$ and $v_{z}$ in terms of $v_{x}$ :

$$
\begin{gathered}
v_{y}^{2}=\left[R^{D}\left(1+R^{\phi}\right)-1\right] v_{x}^{2}, \\
v_{z}^{2}=R^{\phi} v_{x}^{2} .
\end{gathered}
$$

\section{B. Interacting case}

We can now consider the interacting case. We exploit the fact that the operators $\sigma_{i}$ and $\hat{x}$ in the subspace spanned by $\{|0\rangle,|1\rangle\}$ can be written in terms of the $\tau_{i}$ operators [Eqs. (11) and (12)]. We neglect the decay rate from and to the third level, which is small, as it is only due to oscillator damping and vanishes exponentially for $k_{B} T \ll \hbar \omega_{\mathrm{m}}$. Then, we obtain the following results for the decay and decoherence rates of the nanomechanical qubit:

$$
\begin{aligned}
& \Gamma_{1 \rightarrow 0}^{\mathrm{qb}}=2 \pi S_{1}\left(\omega_{10}\right)\left(v_{z}^{2} \beta_{4}^{2}+v_{y}^{2} \beta_{3}^{2}\right)+2 \pi S_{2}\left(\omega_{10}\right) \beta_{5}^{2}, \\
& \Gamma_{01}^{\mathrm{qb}, \phi}=4 \pi S_{1}(0) v_{x}^{2} \beta_{2}^{2} .
\end{aligned}
$$

Using the relations (37) and (38) and assuming that $S_{i}\left(\omega_{10}\right) \approx S_{i}\left(\omega_{\mathrm{m}}\right)$, we find

$$
\begin{aligned}
\Gamma_{1 \rightarrow 0}^{\mathrm{qb}}= & \Gamma_{1 \rightarrow 0}^{\mathrm{c}}(\pi / 2) \frac{R_{\phi} \beta_{4}^{2}+\left[R^{D}\left(1+R^{\phi}\right)-1\right] \beta_{2}^{2}}{1+R^{\phi}} \\
& +\beta_{5}^{2} \gamma\left(1+n_{\mathrm{th}}\right), \\
& \Gamma_{01}^{\mathrm{qb}, \phi}=\beta_{2}^{2} \Gamma_{01}^{\mathrm{c}, \phi}(\pi / 2) .
\end{aligned}
$$

In the region of interest, we can use the analytical expressions for $\beta_{i}$. For $\hbar \omega_{\mathrm{m}} / t \ll 1$, we can drop the term proportional to $\beta_{2}^{2} \ll \beta_{4}^{2}$ and obtain

$$
\begin{aligned}
\Gamma_{1 \rightarrow 0}^{\mathrm{qb}} \approx & \frac{R_{\phi}}{1+R^{\phi}} \frac{4 \hbar^{2} g^{2} t^{2} \omega_{\mathrm{m}}}{\Delta^{4} \omega_{\mathrm{m}}^{\prime}} \Gamma_{1 \rightarrow 0}^{\mathrm{c}}(\pi / 2) \\
& +\frac{\omega_{\mathrm{m}}}{\omega_{\mathrm{m}}^{\prime}}\left[1-\frac{4 \hbar^{3} g^{2} t \omega_{\mathrm{m}}}{\Delta^{4}}\right] \gamma\left(1+n_{\mathrm{th}}\right) .
\end{aligned}
$$

The pure dephasing is controlled by $\beta_{2}^{2} \approx(\hbar g / t)^{4} \ll 1$. The dephasing is thus strongly reduced in the nanomechanical qubit in comparison to the charge system.

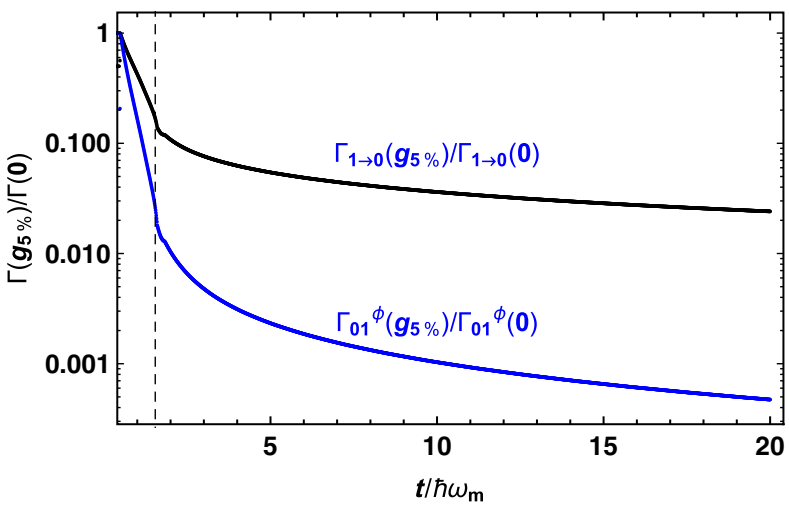

FIG. 9. Ratio of the decay rate $\Gamma_{1 \rightarrow 0}^{\mathrm{qb}}\left(g_{5 \%}\right) / \Gamma_{1 \rightarrow 0}^{\mathrm{qb}}(g=0)$ and pure decoherence rate $\Gamma_{10}^{\mathrm{qb}, \phi}\left(g_{5 \%}\right) / \Gamma_{10}^{\mathrm{qb}, \phi}(g=0)$ as a function of $t / \hbar \omega_{\mathrm{m}}$. We assume $R^{D}=R^{\phi}=1$, and we neglect oscillator damping $(\gamma=0)$. The vertical dashed line indicates the beginning of the region where the qubit becomes dominated by the two charge states, i.e., where $t<1.54 \hbar \omega_{\mathrm{m}}$ (cf. also inset of Fig. 4).

We can numerically evaluate the reduction of the decay and pure-dephasing rates for the case $R^{D}=R^{\phi}=1$. The result for $\Gamma_{1 \rightarrow 0}^{\mathrm{qb}}\left(g_{5 \%}\right) / \Gamma_{1 \rightarrow 0}^{\mathrm{qb}}(g=0)$ and $\Gamma_{10}^{\mathrm{qb}, \phi}\left(g_{5 \%}\right) /$ $\Gamma_{10}^{\mathrm{qb}, \phi}(g=0)$ is shown in Fig. 9 as a function of $t$ for $\gamma=0$. As expected from the analytical expressions, the larger the value of $t$, the larger the reduction in the decoherence, which is a natural consequence of the mechanical nature of the qubit in this limit.

\section{TWO-QUBIT GATE}

We have shown that a carbon-nanotube oscillator can be used as a qubit and how manipulation and readout can be performed. To use these devices to manipulate quantum information, an entangling two-qubit gate is required. In this section, we discuss a possible implementation of the CNOT gate, known to be a universal gate. We follow the idea presented in Ref. [49] that exploits the coupling of two superconducting qubits to the same microwave cavity and that has been successfully implemented as reported in Ref. [50].

We consider the effective coupling generated by a microwave cavity between two nanomechanical qubits. In the case of qubits that can be well approximated as two-level systems, the coupling to the cavity is of the form of Eq. (20): $\hbar g_{v}^{(a)} \tau_{x}^{(a)}\left(b+b^{\dagger}\right)$, where the index $a$ takes the value 1 or 2 to indicate the two qubits. One can show that this induces a coupling term in the Hamiltonian, $J \tau_{x}^{(1)} \tau_{x}^{(2)}$. The driving of the first qubit at the resonant frequency of the second qubit can be described by a Hamiltonian term $\hbar A \cos \left(\omega_{D} t\right) \tau_{x}^{(1)}$, where $A$ is the intensity and $\omega_{D}=\omega_{10}^{(2)}$ the driving frequency. Taking into account the effective coupling induced between the two qubits, this translates into the term $\hbar J_{z x} \tau_{z}^{(1)} \tau_{x}^{(2)}$ in the rotating frame Hamiltonian, with 


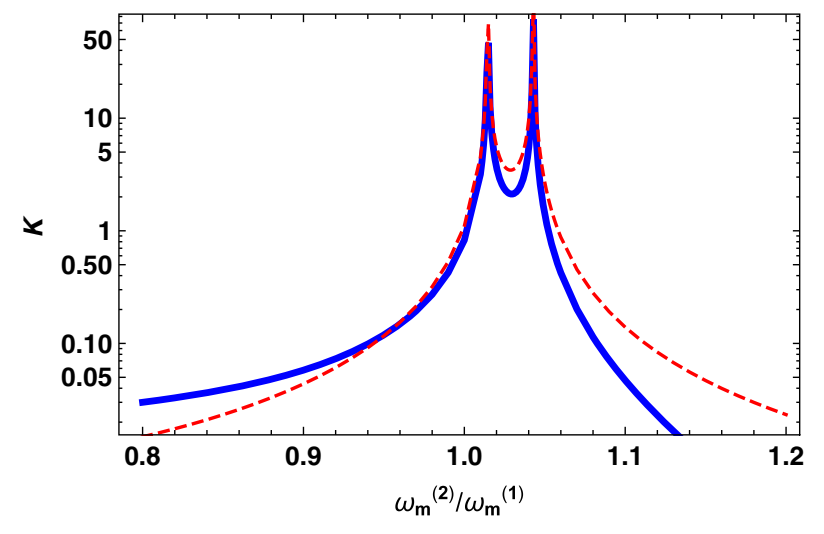

FIG. 10. Coefficient $K$ of the contributions to $J_{z x}$ that diverge like $\left.\omega_{\mathrm{m}}^{(1)} /\left(\omega_{c}-\omega_{10}\right)^{(2)}\right)$ [divided by the two coupling constants and the driving intensity $\left.g_{\mathrm{ec}}^{(1)} g_{\mathrm{ec}}^{(2)} A\right]$ as a function of $\omega_{\mathrm{m}}^{(2)}$. The solid line is the numerical result, and the dashed line is the analytical one, Eq. (43). The other parameters are $g^{(1)}=g^{(2)}=1.264 \hbar \omega_{\mathrm{m}}^{(1)}$, $t^{(1)}=10 \hbar \omega_{\mathrm{m}}^{(1)}$, and $t^{(2)}=10.5 \hbar \omega_{\mathrm{m}}^{(1)}$.

$$
J_{z x}=\frac{4 g_{v}^{(1)} g_{v}^{(2)} A \omega_{c} \omega_{10}^{(1)}}{\left(\omega_{10}^{(1)^{2}}-\omega_{10}^{(2) 2}\right)\left(\omega_{c}^{2}-\omega_{10}^{(2) 2}\right)}
$$

This term is the required gate generating function leading to the evolution operator $e^{-i t_{J} J_{z x} \tau_{z}^{(1)} \tau_{x}^{(2)}}=\cos \left(J_{z x} t_{J}\right)-$ $i \sin \left(J_{z x} t_{J}\right) \tau_{z}^{(1)} \tau_{x}^{(2)}$, that allows the CNOT gate to be performed, modulo single-qubit rotations, in a time $t_{J}=\pi / 2 J_{z x}$.

Thus, one expects that this operation can be applied to the mechanical qubits, but since the anharmonicity is not very large, we need to investigate the contributions of the higher lying states. We proceed similarly to what we did for the dispersive coupling in Sec. IV F. A perturbative calculation is described in Appendix C. It gives

$$
J_{z x}=\frac{A g_{\mathrm{ec}}^{(1)} g_{\mathrm{ec}}^{(2)} \beta_{1,4}^{(2)}}{g_{\mathrm{ec}}^{(1) 2}\left(\omega_{c}-\omega_{10}^{(2)}\right)}\left[\chi_{m}^{(1)}\left(\omega_{10}^{(2)}\right)+\chi_{e}^{(1)}\left(\omega_{10}^{(2)}\right)\right]
$$

The expression holds for small $g / t$. We note that the coefficient diverges for $\omega_{c}=\omega_{10}^{(2)}$, while in contrast to what is found for the dispersive coupling, no divergence is present for $\omega_{c}$ close to $\delta_{00}$. We already discussed the functions $\chi_{m}$ and $\chi_{e}^{(1)}$ in Sec. IV F; we note here that $\chi_{e}$ diverges when its argument equals $\delta_{00}^{(1)}$ or $\delta_{11}^{(1)}$. Since, in general, $\omega_{10}^{(2)} \ll \delta_{00}^{(1)} \approx \delta_{11}^{(1)}$, the contribution of $\chi_{e}^{(1)}$ is much smaller than that of $\chi_{m}^{(1)}$, which diverges when its argument equals $\omega_{10}^{(1)}$ and $\omega_{12}^{(1)}$. Figure 10 shows the dependence of the factor $K=J_{z x} \omega_{\mathrm{m}}^{(1)}\left(\omega_{c}-\omega_{10}^{(2)}\right) / g_{\mathrm{ec}}^{(1)} g_{\mathrm{ec}}^{(2)} A$ as a function of the ratio $\omega_{\mathrm{m}}^{(2)} / \omega_{\mathrm{m}}^{(1)}$. Both the exact numerical (solid line) and analytical expression Eq. (43) (dashed line) are shown.
The double peak corresponds to the values for which $\omega_{10}^{(2)}$ equals either $\omega_{10}^{(1)}$ or $\omega_{21}^{(1)}$ [cf. Eq. (22)].

This result shows that by driving qubit 1 , it is possible to induce a time-dependent evolution that generates the CNOT gate.

\section{PROSPECT FOR EXPERIMENTAL IMPLEMENTATION}

The results found in the previous two sections are very promising for the experimental realization of a nanomechanical qubit. In this section, we discuss possible experimental implementations using currently available technology. As discussed in the Introduction, the double quantum dot can be realized in a suspended carbon nanotube and coupled to the second mechanical flexural mode of the nanotube. Such a device has recently been measured at $2 \mathrm{~K}$ [29], reporting values of $t / 2 \pi \hbar=$ 49-96 GHz with a tunable value of $\epsilon$, and a second mechanical mode of frequency $\omega_{\mathrm{m}} / 2 \pi=327 \mathrm{MHz}$ with a mechanical quality factor $Q=4 \times 10^{3}$ and a coupling constant $g / 2 \pi=320 \mathrm{MHz}$. Taking these parameters, we have $t / \hbar \omega_{\mathrm{m}}$ up to $150-300$, and $g / \omega_{\mathrm{m}} \approx 1$, noting that, of course, $t$ can be tuned to lower values. Choosing $t=7 \hbar \omega_{\mathrm{m}}$, we can operate on the $g_{5 \%}$ line (cf. Fig. 4) without changing other parameters. At this value of $g$, we already have a sizable reduction of both the decoherence and decay rates of the mechanical qubit, $\Gamma_{1 \rightarrow 0}$ and $\Gamma_{10}^{\phi}$ [cf. Fig. 9], compared to that of the charge double quantum dot. The experiment at $2 \mathrm{~K}$ realized with a device fabricated on a $\mathrm{Si}$ substrate reports an incoherent tunneling rate $\Gamma_{1 \rightarrow 0}$ estimated to $2 \pi \times 510 \mathrm{MHz}$, which is clearly too large to use for qubit operations. However, improvements should be possible by operating the device at $10 \mathrm{mK}$ to suppress the decoherence induced by low-frequency vibrations (phonon) modes, by producing devices on sapphire substrates that host a minimal number of charge fluctuators, and by current-annealing the nanotube in situ in the dilution fridge to remove all the contamination adsorbed on the surface of the nanotube [51]. Double-dot structures have been created in nonsuspended carbon nanotubes and have been coupled to superconducting cavities [52].

One can thus target a mechanical resonator cooled at $10 \mathrm{mK}$ with $\omega_{\mathrm{m}} / 2 \pi$ in the range of $0.6-1 \mathrm{GHz}$ using a nanotube that is shorter and/or is under mechanical tension. A value of $t / \hbar \omega_{\mathrm{m}}=10$ will then require a coupling constant of the order of $1.1 \hbar \omega_{\mathrm{m}}$, which can be obtained by reducing the nanotube-gate separation and/or increasing the voltage applied on the gate electrode. With these values, the reduction of the pure-dephasing decoherence rate of the mechanical qubit with respect to that of the double quantum dot will be about $10^{3}$. Assuming that the decoherence rate of the order of $3 \mathrm{MHz}$ can be obtained (as was achieved in GaAs double quantum dots [46]) and that it is mainly limited by pure dephasing, it should be possible to implement most of the 
standard protocols for quantum computation using a mechanical qubit with a $3-\mathrm{kHz}$ decoherence rate. Note that we did not consider the decoherence induced by the mechanical damping. Assuming a $Q$ of $10^{6}$, which has been experimentally observed in suspended carbon nanotubes [14], this would give a decoherence rate of only $500 \mathrm{~Hz}$. Another possible implementation consists in using a nonsuspended GaAs double quantum dot with a $3-\mathrm{MHz}$ charge decoherence rate coupled to a suspended metal beam, such as a carbon nanotube.

With these parameters, one could implement a CNOT gate by choosing $\omega_{10}^{(1)} / 2 \pi=500 \mathrm{MHz}$ and $\omega_{10}^{(2)} / 2 \pi=$ $550 \mathrm{MHz}$ (these values are reduced with respect to the oscillator mechanical frequencies), and tune the cavity to $\omega_{c} / 2 \pi=475 \mathrm{MHz}$. For $t^{(a)} / \hbar \omega_{\mathrm{m}}^{(a)} \approx 10$, one obtains $K$ of the order of 1 . We assume a coupling constant $g_{\mathrm{ec}} / 2 \pi=$ $50 \mathrm{MHz}$ of the order as what was reported in Ref. [53] for carbon nanotubes coupled to superconducting cavities. With these values and a drive $A / 2 \pi$ also of the order of $50 \mathrm{MHz}$, which is the detuning between the two qubit frequencies, one finds that $J_{z x} / 2 \pi \approx 2.5 \mathrm{MHz}$, which is of the same order as what was used in Ref. [50] to implement the CNOT gate in superconducting qubits.

With the chosen value of $\omega_{\mathrm{m}}$, the typical range for $\omega_{01} / 2 \pi$ does not exceed $500 \mathrm{MHz}$. This is sufficient to perform single- and two-qubit operations, but error correction could be difficult since a very low level of thermal occupation is required. In the long term, it seems feasible to increase the mechanical frequency to higher values; a qubit splitting of $1 \mathrm{GHz}$ is the target for implementing error correction.

\section{QUANTUM SENSING OF A STATIC FORCE WITH THE NANOMECHANICAL QUBIT}

As an important application, we discuss here the possibility of using the nanomechanical qubit for quantum sensing. A mechanical oscillator can couple to a variety of forces; independently of the nature of the force, the additional term in the Hamiltonian describing this coupling can be written as $H_{F}=F x$, with $F$ the external force. In terms of the nanomechanical qubit operators, this gives $H_{F}=F \gamma_{F} \tau_{x} / 2$, with $\gamma_{F}=2 x_{\mathrm{z}} \beta_{5}$ [cf. Eq. (12); we introduced a factor of 2 for convenience in the notation]. One can then use the protocols for qubit preparation and readout in order to measure $F$ with great sensitivity.

As a relevant example, we consider here the Rabi measurement protocol, as described in Sec. IV D of Ref. [13]. In a nutshell, it consists in preparing the qubit in the ground state and then letting it evolve in the presence of the static force $F$ according to the Hamiltonian

$$
H=\hbar\left(\omega_{10} \tau_{z}+\omega_{F} \tau_{x}\right) / 2,
$$

with $\omega_{F}=F \gamma_{F} / \hbar$. This process induces a Larmor-like precession with a Rabi frequency $\omega_{\mathrm{R}}=\sqrt{\omega_{10}^{2}+\omega_{F}^{2}}$ of the pseudospin representing the qubit state in the Bloch sphere around the direction of the effective magnetic field vector $\left(\omega_{F}, 0, \omega_{10}\right)$. The probability $P_{1}$ of measuring the qubit in the excited state oscillates as

$$
P_{1}=\frac{\omega_{F}^{2}}{\omega_{\mathrm{R}}^{2}} \sin ^{2}\left(\omega_{\mathrm{R}} t / 2\right)
$$

For large $t$, the sine part of the expression is very sensitive to a small variation of $\omega_{\mathrm{R}}$ and thus of the force. For a detection time $t_{d}$ such that $\omega_{\mathrm{R}} t_{d}=\pi / 2+k \pi$, with $k$ a large integer, one finds

$$
\delta P_{1} \approx\left(\frac{\omega_{F}}{\omega_{\mathrm{R}}}\right)^{3} \frac{\gamma_{F} t_{d}}{2 \hbar} F
$$

The sensitivity thus increases with the oscillation time $t_{d}$. This is mainly limited by the coherence time of the qubit. One also sees that in order to have a large signal, it is better to have $\omega_{F}$ of the same order or larger than $\omega_{10}$. In our case, this could be achieved using the gate voltage that generates an additional, controllable static force to the oscillator. The most fundamental source of uncertainty in quantum sensing is the binomial fluctuation of the qubit readout outcome. Following Ref. [13], a rough estimate of the signal-to-noise ratio that can be achieved with this method gives the minimum detectable static force per unit bandwidth as

$$
\delta F_{\min } \approx \frac{\hbar}{\gamma_{F} \sqrt{T_{2}}}
$$

where $T_{2}$ is the coherence time. Using typical values for carbon nanotube resonators $\omega_{\mathrm{m}}=2 \pi \times 600 \mathrm{MHz}$, $m=10^{-21} \mathrm{Kg}$, one has $x_{\mathrm{z}} \approx 4 \times 10^{-12} \mathrm{~m}$. Using $T_{2} \sim$ $50 \mu$ s from the $3-\mathrm{kHz}$ decoherence rate for the nanotube mechanical qubit estimated in the last section, the staticforce sensitivity is about $10^{-21} \mathrm{~N} / \mathrm{Hz}^{1 / 2}$. For comparison, the resolution in static-force measurements is $10^{-17} \mathrm{~N}$ using optically levitated particles [54] and $10^{-12} \mathrm{~N}$ with atomicforce cantilevers in high vacuum and at low temperatures [55], while a sensitivity of $10^{-15} \mathrm{~N} / \mathrm{Hz}^{1 / 2}$ can be achieved using optical tweezers in liquids [56]. One finds that when the electronic contribution to the decoherence is neglected with respect to the mechanical part, then quantum sensing can reach sensitivities of the order of the standard quantum limit [57].

\section{CONCLUSIONS}

In conclusion, we have shown that coupling a double quantum dot capacitively to the second flexural mode of a suspended carbon nanotube, and appropriately tuning the hopping amplitude between the two charge states of the quantum dot, one can introduce a strong anharmonicity in the spectrum of the mechanical mode. This approach 
enables one to directly address the first two energy quasimechanical eigenstates without populating the third state (cf. Fig. 4). These two states form a qubit with mainly a mechanical character. Manipulation and readout are then possible with standard techniques, but at the same time, we found that the coupling to the environment is strongly reduced. The main benefit is the reduction by up to 3-4 orders of magnitude of the pure-dephasing rate, with respect to the double quantum dot. Combined with the expectation of improved dephasing times, this suggests the potential for nanomechanical qubits with very long coherence times. Furthermore, the production of mechanical devices using conventional microfabrication techniques is promising for scalability.

The mechanical qubit can be used to couple to a wide number of modalities for external fields, including acceleration, magnetic forces, or other forces. We have shown that any fields that induce forces on the mechanical oscillator can be detected with unprecedented sensitivity, using quantum preparation and detection protocols.

We have shown that the nanomechanical qubits can be coupled to each other by microwave cavities, allowing the implementation of a CNOT gate with purely microwave control. In principle, all other operations involving multiple qubits can be obtained by applying the CNOT gate and single-qubit operations.

On the more technical side, we also found a unitary transformation, valid in the dispersive limit of $g / \mid t / \hbar-$ $\omega_{\mathrm{m}} \mid \ll 1$, that maps the problem to the anharmonic oscillator, giving the explicit expressions of the main physical operators in the qubit subspace.

\section{ACKNOWLEDGMENTS}

F. P. acknowledges support from the French Agence Nationale de la Recherche (Grant No. SINPHOCOM ANR-19-CE47-0012) and Idex Bordeaux (Maesim Risky project 2019 of the LAPHIA Program). A. N. C. acknowledges support from the Air Force Office of Scientific Research, the Army Research Laboratory, the DOE, Office of Basic Energy Sciences, and from the University of Chicago MRSEC (NSF Grant No. DMR-1420709). A. B. acknowledges ERC Advanced Grant No. 692876, AGAUR (Grant No. 2017SGR1664), MICINN Grant No. RTI2018097953-B-I00, the Fondo Europeo de Desarrollo, the Spanish Ministry of Economy and Competitiveness through the "Severo Ochoa" program for Centres of Excellence in R\&D (CEX2019-000910-S), Fundacio Privada Cellex, Fundacio Mir-Puig, and Generalitat de Catalunya through the CERCA program.

\section{APPENDIX A: ELECTROSTATICS AND DERIVATION OF THE COUPLING CONSTANTS}

Here, we give a derivation of the Hamiltonian. Thus, we need to calculate the electrostatic energy of the system.
The only subtle point is the contribution of the voltage sources, as is well known for the Coulomb blockade problem [58]. One needs the electrostatic energy as a function of the charges in the system and not of the voltages, which is particularly important for the expression of the mechanical force. Following Ref. [34] (Appendix A), the electrostatic problem of $N$ conductors plus a ground conductor can be treated by introducing a capacitance matrix $C_{i j}^{(0)}$ for which the charges on the conductor $i$ can be related to the potentials of the other conductors:

$$
Q_{i}=\sum_{j=0}^{N} C_{i j}^{(0)} V_{j} .
$$

Here, $C_{i i}^{(0)}=\sum_{i \neq j} c_{i j}$ and $C_{i j}^{(0)}=-c_{i j}$, where $c_{i j}$ is the capacitance between conductor $i$ and $j$ and, clearly, ${ }^{t} C=C$. In the list of conductors, we include the ground with the index 0 . The relation given by Eq. (A1) cannot be inverted since the capacitance matrix has a vanishing determinant, which just indicates that one can shift all the potential by a constant. One can then set one of the potentials to 0 , say, the ground, and eliminate one line of the matrix, which we choose to be that related to the charge on the ground. The $N \times N$ capacitance matrix obtained in this way, $C_{i j}$, is then invertible, and one can write

$$
V_{i}=\sum_{j=1}^{N}\left(C^{-1}\right)_{i j} Q_{j} .
$$

The total energy of the system is $U=\sum_{i=0}^{N} V_{i} Q_{i} / 2$. With our choice of $V_{0}=0$, it reduces to $U=\sum_{i=1}^{N} V_{i} Q_{i} / 2=$ ${ }^{t} V Q / 2$, where we introduced the vector notation for the charge and the potentials. Using the capacitance matrix, we have

$$
U=\frac{1}{2}{ }^{t} V C V=\frac{1}{2}{ }^{t} Q C^{-1} Q .
$$

In typical problems, one needs to include potential sources. These sources can be modeled with metallic leads with a macroscopic capacitance to the ground $C_{B} \rightarrow \infty$, and the charge on this island $Q_{B} \rightarrow \infty$ with $Q_{B} / C_{B}=V_{B}$ constant. In the following, without loss of generality, we assume that the capacitances of all sources have the same value $C_{B}$.

The relevant energy for the problem at hand is the energy expressed as a function of the charges in the metallic islands and leads. The mechanical displacement $x$ of any mechanical element of the circuit induces a change in the capacitance matrix, which acquires a dependence on the displacement $C(x)$. (For simplicity, we consider a single mechanical mode whose displacement is parametrized by the variable $x$; generalization to several modes is straightforward.) 
The expression for the potential energy is thus

$$
U(Q, x)=\frac{1}{2}^{t} Q C(x)^{-1} Q .
$$

From this expression, we can find the expression of the potential energy as a function of the charges in the dots and $x$. We can then eliminate the charges in the leads by using their potentials. For this purpose, we need to invert the matrix $C$, exploiting the large $C_{B}$ limit. Following Ref. [34], we first divide the indices in $c$ and $v$, for charge nodes and voltage sources, respectively. We can write

$$
C=\left(\begin{array}{ll}
C_{c c} & C_{c v} \\
C_{v c} & C_{v v}
\end{array}\right)
$$

The inverse of this matrix can be written as follows:

$$
\begin{gathered}
\left(C^{-1}\right)_{c c}=C_{c c}^{-1}+C_{c c}^{-1} C_{c v} D C_{v c} C_{c c}^{-1}, \\
\left(C^{-1}\right)_{v c}=-D C_{v c} C_{c c}^{-1}, \\
\left(C^{-1}\right)_{v v}=C_{v v}^{-1}\left(1-C_{v c} C_{c c}^{-1} C_{c v} D\right),
\end{gathered}
$$

where $D=\left(C_{v v}-C_{v c} C_{c c}^{-1} C_{c v}\right)^{-1}$. Since we eliminated the ground metal island, the only macroscopic matrix elements left are in the diagonal part of $C_{v v} \sim C_{B}$ [cf. Eq. (A17) in the following]. We can then greatly simplify the inverse since, to leading order in $C_{B}$, one has $D=1 / C_{B}$,

$$
\begin{gathered}
\left(C^{-1}\right)_{c c}=C_{c c}^{-1}, \\
\left(C^{-1}\right)_{v c}=-C_{v c} C_{c c}^{-1} / C_{B}, \\
\left(C^{-1}\right)_{v v}=1 / C_{B} .
\end{gathered}
$$

This result allows us to express the energy as follows:

$U=\frac{1}{2}{ }^{t} Q_{c} C_{c c}^{-1} Q_{c}-{ }^{t} Q_{c} C_{c c}^{-1} C_{c v} Q_{v} / C_{B}+{ }^{t}{ }^{t} Q_{v} Q_{v} / C_{B}$,

but $Q_{v} / C_{B}=V_{v}$ are the source voltages and the last term is independent of $Q_{c}$. We thus have

$$
U=\frac{1}{2}{ }^{t} Q_{c} C_{c c}^{-1} Q_{c}-{ }^{t} Q_{c} C_{c c}^{-1} C_{c v} V_{v}
$$

\section{Couplings}

From this expression, we can derive the coupling to the mechanical displacement and to the voltage applied to a nearby gate electrode. For this purpose, we include the $x$ dependence of the capacitances and the substitution $V_{v}=V_{v}^{D C}+V_{v}^{\mathrm{ac}}$, where $V_{v}^{D C}$ is the static part and $V_{v}^{\mathrm{ac}}$ the oscillating part of the voltage. If a gate electrode is part of an electromagnetic cavity, one can obtain the coupling to the photon creation and destruction operators via the substitution $V_{v}^{\text {ac }}=V_{v}^{\mathrm{z}}\left(b_{v}+b_{v}^{\dagger}\right)$, where $V_{v}^{\mathrm{z}}$ is the zero-point voltage of the cavity and $b_{v}$ the destruction operator for the photons.

We now need a description in terms of the charge fields. Let us associate to each charge variation $\delta q_{c}^{i}$ the occupation operator $n_{i}$ with eigenvalues 0 or 1 so that the operator for the total number of charges can be written as $Q_{c}=Q_{c}^{0}+\sum_{i} n_{i} \delta q_{c}^{i}$. The index $i$ can take into account spin or other degrees of freedom, and we include a background frozen charge $Q_{c}^{0}$. By including this expression in Eq. (A13), at lowest order in $x$, we obtain

$$
\begin{aligned}
U= & U_{C}+x \sum_{i} n_{i}\left(\lambda_{i}^{\mathrm{em}}+\sum_{j \neq i} n_{j} \lambda_{i j}^{\mathrm{em}}\right) \\
& +\sum_{i} n_{i} \lambda_{i v}^{\mathrm{ev}} V_{v}^{A C}+x \lambda_{v}^{\mathrm{mv}} V_{v}^{A C},
\end{aligned}
$$

where

$U_{C}=\sum_{i} n_{i}{ }^{t} \delta q_{c}^{i} C_{c c}^{-1}\left(Q_{c}^{0}-C_{c v} V_{v}^{D C}+\sum_{j} \frac{1}{2} \delta q_{c}^{j} n_{j}\right)$

is the pure Coulomb part and the other three terms describe the interaction between the 3 degrees of freedom, $x, V^{a c}$, and $n_{i}$, which are associated with the indices $m, v$, and $e$, respectively. (We discarded the constant $U_{0}={ }^{t} Q_{c}^{0} C_{c c}^{-1} Q_{c}^{0} / 2-{ }^{t} Q_{c}^{0} C_{c c}^{-1} C_{c v} V_{v}^{D C}$.) Here,

$\lambda_{i}^{\mathrm{em}}=\partial_{x}\left[\left({ }^{t} Q_{c}^{0}+\frac{{ }^{t} \delta q_{c}^{i}}{2}\right) C_{c c}^{-1}-V_{v}^{D C} C_{v c} C_{c c}^{-1}\right] \delta q_{c}^{i}$

and $\lambda_{i j}^{\mathrm{em}}={ }^{t} \delta q_{c}^{i} \partial_{x} C_{c c}^{-1} \delta q_{c}^{j} / 2$ are the electromechanical couplings, $\lambda_{i v}^{\mathrm{ev}}=-{ }^{t} \delta q_{c}^{i} C_{c c}^{-1} C_{c v}$ the voltage-electron coupling, and $\lambda_{v}^{\mathrm{mv}}=-{ }^{t} Q_{c}^{0} \partial_{x}\left(C_{c c}^{-1} C_{c v}\right)$ the mechanical oscillatorvoltage coupling.

\section{Single- and double-dot cases}

We now consider two examples.

Single dot.-In this case, we have four metallic entities: one for the dot; three for the left, right, and gate leads [cf. Fig. 11(a)]. The matrix $C$ reads

$C=\left(\begin{array}{cccc}C_{1} & -C_{R} & -C_{L} & -C_{g} \\ -C_{R} & C_{B}+C_{R} & 0 & 0 \\ -C_{L} & 0 & C_{B}+C_{L} & 0 \\ -C_{g} & 0 & 0 & C_{B}+C_{g}\end{array}\right)$,

with obvious notation for the capacitances and with $C_{1}=C_{L}+C_{R}+C_{g}$. From this expression one obtains 
(a)

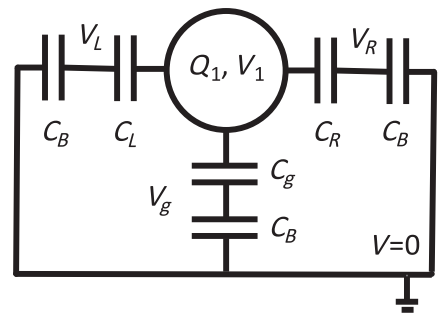

(b)

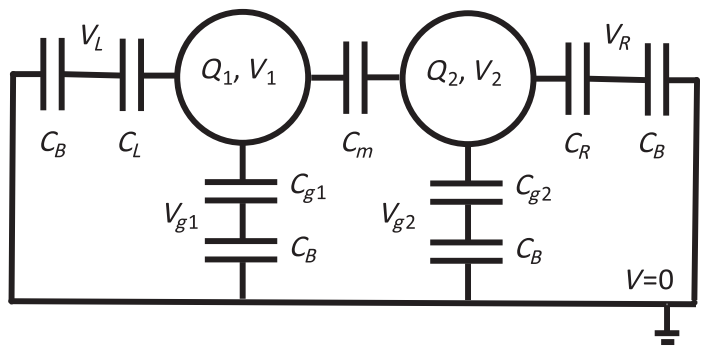

FIG. 11. Network of capacitances representing the (a) singleand (b) double-dot circuits. The capacitances $C_{B}$ are used to model the voltage sources.

$C_{c c}=C_{1}, \quad C_{c v}=-\left(C_{R}, C_{L}, C_{g}\right), \quad$ and $\quad C_{v v}=C_{B}+$ $\operatorname{diag}\left(C_{R}, C_{L}, C_{g}\right)$. We assume that only $C_{g}$ depends on $x$, which gives $\partial_{x} C_{c c}=\partial_{x} C_{g}=C_{g}^{\prime}$ and $\partial_{x} C_{c v}=$ $-C_{g}^{\prime}(0,0,1)$. We also have $\delta q_{c}^{i}=-e$ (with $e$ the electron charge), and for simplicity, we report the expressions for $V_{L}=V_{R}=0$. We then have, for the couplings,

$$
\lambda_{i}^{\mathrm{em}}=e C_{g}^{\prime}\left[Q_{0}-\left(C_{1}-C_{g}\right) V_{g}-e / 2\right] / C_{1}^{2},
$$

$\lambda_{i j}^{\mathrm{em}}=-e^{2} C_{g}^{\prime} /\left(2 C_{1}^{2}\right)$, and $\lambda_{v}^{\mathrm{mv}}=-C_{g}^{\prime} Q_{0}\left(C_{R}, C_{L}, C_{g}-C_{1}\right) /$ $C_{1}^{2}$. The last coupling constant is related to $\lambda^{\mathrm{em}}$. Using the value of $Q_{0}$ that minimizes the electrostatic energy, $Q_{0}=-C_{g} V_{g}$, and assuming $\left|Q_{0}\right| \gg e$, one obtains, for the single-dot coupling constant, $\lambda_{i}^{\mathrm{em}}=-e C_{g}^{\prime} V_{g} / C_{1}$. Note that, in this limit, we also have $\lambda_{i j}^{e m} / \lambda_{i}^{e m}=e / 2 C_{1} V_{g} \ll 1$.

Double dot.-Let us consider a double dot, with each dot coupled to a gate voltage [cf. Fig. 11(b)]. The capacitance matrix is

$$
\begin{gathered}
C_{c c}=\left(\begin{array}{cc}
C_{1} & -C_{m} \\
-C_{m} & C_{2}
\end{array}\right), \\
C_{c v}=-\left(\begin{array}{cccc}
C_{L} & C_{g 1} & 0 & 0 \\
0 & 0 & C_{g 2} & C_{R}
\end{array}\right),
\end{gathered}
$$

and $C_{v v}=C_{B}+\operatorname{diag}\left(C_{L}, C_{g 1}, C_{g 2}, C_{R}\right)$. Here, $C_{1}=$ $C_{L}+C_{m}+C_{g 1}$ and $C_{2}=C_{R}+C_{m}+C_{g 2}$. We can distinguish two types of $n$ operators: one for dot $1\left(n_{1}\right)$ and the other for dot $2\left(n_{2}\right)$. We have $\delta q_{c}^{1}=(-e, 0)$ and $\delta q_{c}^{2}=(0,-e)$. For simplicity, in the following, we assume a symmetric situation $C_{L}=C_{R}=C, \quad V_{L}=V_{R}=0$, $C_{1}=C_{2}=C_{S}, \quad Q_{c}^{0}=\left(Q_{0}, Q_{0}\right), \quad$ and $\quad V_{v}=\left(0, V_{g 1}\right.$, $\left.V_{g 2}, 0\right)$. For our specific problem, for which the interesting mechanical mode is the second one, we assume that $C_{g 1}(x)=C_{g 2}(-x)$ by symmetry, so $C_{g 1}^{\prime}=-C_{g 2}^{\prime}$. With this hypothesis, we find for the coupling constants,

$$
\begin{aligned}
\lambda_{1}^{\mathrm{em}}+\lambda_{2}^{\mathrm{em}} & =-\frac{e C_{g}^{\prime}\left(C+2 C_{m}\right)\left(V_{g 1}-V_{g 2}\right)}{C_{S}^{2}-C_{m}^{2}}, \\
\lambda_{1}^{\mathrm{em}}-\lambda_{2}^{\mathrm{em}} & =\frac{e C_{g}^{\prime}\left[2 Q_{0}-e-C\left(V_{g 1}+V_{g 2}\right)\right]}{C_{S}^{2}-C_{m}^{2}}, \\
\lambda_{1 v}^{\mathrm{ev}} & =-e \frac{\left(C C_{S}, C_{g} C_{S}, C_{g} C_{m}, C C_{m}\right)}{C_{S}^{2}-C_{m}^{2}}, \\
\lambda_{2 v}^{\mathrm{ev}} & =-e \frac{\left(C C_{m}, C_{g} C_{m}, C_{g} C_{S}, C C_{S}\right)}{C_{S}^{2}-C_{m}^{2}}, \\
\lambda_{v}^{\mathrm{mv}} & =Q_{0} C_{g}^{\prime} \frac{\left(-C, 2 C_{m}+C,-2 C_{m}-C, C\right)}{C_{S}^{2}-C_{m}^{2}},
\end{aligned}
$$

and $\lambda_{12}^{\mathrm{em}}=0$. For $V_{g 1}=V_{g 2}=V_{g}, \lambda_{1}^{\mathrm{em}}=-\lambda_{2}^{\mathrm{em}}=\lambda^{\mathrm{em}}$, leading to the Hamiltonian term that we used in the main text: $\lambda^{\mathrm{em}} x\left(n_{1}-n_{2}\right)$. When we reduce the Hilbert space to the two charge states $(1,0)$ and $(0,1)$, this Hamiltonian term can be written as $\lambda^{\mathrm{em}} x \sigma_{z} \equiv-\hbar g\left(x / x_{\mathrm{z}}\right) \sigma_{z}$. In this basis, $n_{1}=\left(\sigma_{z}+\mathbb{1}\right) / 2$ and $n_{2}=\left(\mathbb{1}-\sigma_{z}\right) / 2$, which gives

$$
g=\frac{e C_{g}^{\prime}\left[2 C V_{g}+e-2 Q_{0}\right] x_{z}}{2 \hbar\left(C_{S}^{2}-C_{m}^{2}\right)} .
$$

For the case $Q_{0}=-C_{g} V_{g},\left|Q_{0}\right| \gg e$, and $C_{m} \ll C_{S}$, we obtain $g=e C_{g}^{\prime} V_{g} x_{z} / \hbar C_{S}$, which coincides with the singledot coupling constant. We also have the coupling of the charge of the dots to the voltages of the gate electrodes:

$$
H^{\mathrm{ev}}=\frac{e C_{g}}{2}\left[1 \frac{V_{g 1}^{a c}+V_{g 2}^{a c}}{C_{S}-C_{m}}+\sigma_{z} \frac{V_{g 1}^{a c}-V_{g 2}^{a c}}{C_{S}+C_{m}}\right] .
$$

Finally, the direct coupling between the mechanical oscillator and the voltages of the gate electrodes is

$$
H^{\mathrm{mv}}=Q_{0} C_{g}^{\prime} \frac{C+2 C_{m}}{C_{S}^{2}-C_{m}^{2}} x\left(V_{g 1}^{a c}-V_{g 2}^{a c}\right) .
$$

In order to compare the last two coupling constants, we can write this part of the Hamiltonian as follows:

$$
H=\left[\lambda^{\mathrm{ev}} \sigma_{z}+\lambda^{\mathrm{mv}} x_{\mathrm{z}}\left(a+a^{\dagger}\right)\right] V_{g 12}^{a c},
$$

with $\lambda^{\mathrm{ev}}=e C_{g} /\left[2\left(C_{S}+C_{m}\right)\right], \quad \lambda^{\mathrm{mv}}=Q_{0} C_{g}^{\prime}\left(C+2 C_{m}\right) /$ $\left(C_{S}^{2}-C_{m}^{2}\right), V_{g 12}^{a c}=V_{g 1}^{a c}-V_{g 2}^{a c}$, and we use $x=x_{\mathrm{z}}\left(a+a^{\dagger}\right)$. 
The ratio of the two coupling constants is then of the order of

$$
\frac{\lambda^{\mathrm{mv}} x_{\mathrm{z}}}{\lambda^{\mathrm{ev}}}=\frac{Q_{0}}{e} \frac{C_{g}^{\prime}}{2 C_{g}} x_{\mathrm{z}} \frac{C+2 C_{m}}{C_{S}-C_{m}} .
$$

In general, this ratio is small, about $\left(Q_{0} / e\right)\left(x_{\mathrm{z}} / L\right)$, where $L=C_{g} / C_{g}^{\prime}$ is typically of the order of the distance of the nanotube from the gate. Thus, the oscillating voltage field couples mainly to the charge degree of freedom.

\section{APPENDIX B: MAPPING OF THE HAMILTONIAN ON THE ANHARMONIC OSCILLATOR IN THE DISPERSIVE REGIME}

In this appendix, we show that the Hamiltonian for the system we are considering given by Eq. (1) can be mapped in the dispersive regime on the Hamiltonian of an anharmonic oscillator. We begin by considering $H$ for $\epsilon=0$. It reduces to $H=t \sigma_{x} / 2+\hbar \omega_{\mathrm{m}} a^{\dagger} a-\hbar g\left(a+a^{\dagger}\right) \sigma_{z}$. Performing a rotation of $\pi / 2$ around the $y$ axis in the charge space with the operator $U_{r}=e^{-i \pi \sigma_{y} / 4}=\left(1-i \sigma_{y}\right) / \sqrt{2}$, one has that $U_{r}^{\dagger} \sigma_{x} U_{r}=\sigma_{z}$ and $U_{r}^{\dagger} \sigma_{z} U_{r}=-\sigma_{x}$, with $\sigma_{y}$ left unchanged. The Hamiltonian is then in the standard form for the Rabi model:

$$
H_{1}=U_{r}^{\dagger} H U_{r}=\frac{t}{2} \sigma_{z}+\hbar \omega_{\mathrm{m}} a^{\dagger} a+\hbar g\left(a+a^{\dagger}\right) \sigma_{x} .
$$

This model has a long history describing the coupling of electromagnetic radiation to a two-level system, but only very recently has it been diagonalized analytically [59]. In practice, it is difficult to make use of this solution, but for the case considered in the present paper, an approximate solution, which holds in the so-called dispersive limit of $\left|t-\hbar \omega_{\mathrm{m}}\right| \ll g$, could be sufficient to obtain an accurate description of the system. As described in Ref. [60], a unitary transformation $D_{1}$ exists such that

$H_{2}=D_{1}^{\dagger} H_{1} D_{1}=t \frac{\sigma_{z}}{2}+\frac{\hbar \omega_{\mathrm{m}}}{4}\left(\hat{p}^{2}+\hat{x}^{2}\right)+\sigma_{z} \hat{x}^{2} \frac{t \hbar g^{2}}{\Delta^{2}}+\ldots$

where we recall $\Delta^{2}=t^{2}-\left(\hbar \omega_{\mathrm{m}}\right)^{2}, \hat{x}=a^{\dagger}+a$, and $\hat{p}=i\left(a^{\dagger}-a\right)$, with $[\hat{x}, \hat{p}]=2 i$. The Hamiltonian is quadratic in $\hat{x}$ and $\hat{p}$ and commutes with $\sigma_{z}$. It can thus be diagonalized as

$$
H_{2}=t \sigma_{z} / 2+\sum_{\sigma= \pm}\left[\hbar \omega_{\sigma} \pi_{\sigma}\left(1 / 2+a_{\sigma}^{\dagger} a_{\sigma}\right)\right]
$$

where $\hat{x}=\sum_{\sigma} \xi_{\sigma}\left(a_{\sigma}^{\dagger}+a_{\sigma}\right) \pi_{\sigma}, \quad \hat{p}=\sum_{\sigma} \xi_{\sigma}^{-1} i\left(a_{\sigma}^{\dagger}-a_{\sigma}\right) \pi_{\sigma}$,

with

$$
\omega_{\sigma}=\omega_{\mathrm{m}}\left[1+4 \sigma t \hbar g^{2} / \omega_{\mathrm{m}} \Delta^{2}\right]^{1 / 2}
$$

the mechanical frequency of each branch, $\pi_{\sigma}=\left(1+\sigma \sigma_{z}\right) / 2$ the projector on the $\sigma$ branch, $\xi_{\sigma}=\left(\omega_{\mathrm{m}} / \omega_{\sigma}\right)^{1 / 2}$, and $\left[a_{\sigma}, a_{\sigma^{\prime}}^{\dagger}\right]=\delta_{\sigma, \sigma^{\prime}}$. Note that this result reduces to the BornOppenheimer picture for $\hbar \omega_{\mathrm{m}} / t \rightarrow 0$. It describes two harmonic oscillators, with different resonating frequencies, the lower branch being softened and the upper being hardened by the interaction.

The transformation found in Ref. [60] allows us to simplify the Hamiltonian only at order 2 in $\hbar g /\left|t-\hbar \omega_{\mathrm{m}}\right|$. For our purposes, we need a transformation allowing us to obtain the form of the Hamiltonian up to the quartic terms in $\hat{x}$. For this reason, we look for a higher-order unitary transformation $D$ that allows us to map $H_{1}$ to $H_{T}=D^{\dagger} H_{1} D$ (the full unitary transformation acting on $H$ includes the rotation $U=U_{r} D$ ), with $H_{T}$ given by Eq. (5) of the main text, valid at order 4 in $g /\left(t / \hbar-\omega_{\mathrm{m}}\right)$.

In general, one can express any unitary transformation as $D=e^{A}$, where $A=-A^{\dagger}$. We begin by expressing the transformation of Ref. [60] in terms of the operators $\hat{x}$ and $\hat{p}$ :

$$
A_{1}=\frac{i \hbar g}{\Delta^{2}}\left(t \sigma_{y} x+\hbar \omega_{\mathrm{m}} \sigma_{x} p\right)
$$

The transformed operators can be found using the standard relation:

$$
e^{A} O e^{-A}=\sum_{n} \frac{1}{n !} C_{n}^{O},
$$

with $C_{n}^{O}=\left[A, C_{n-1}^{O}\right]$ and $C_{0}^{O}=O$. Performing the expansion at order 2 for $O=H_{1}$ and $A=A_{1}$, one obtains the expression for $\mathrm{H}_{2}$. Performing the expansion at order 4 generates the sought-after terms $x^{4}$, but also other terms proportional to $x^{3} \sigma_{x}, x p x \sigma_{y}$, and $x^{2} p^{2} \sigma_{z}$. In order to eliminate these terms, we add two terms to the $A_{1}$ operator so that $A=A_{1}+g^{3} A_{3}+g^{4} A_{4}$. By inspection of the terms generated, one can realize that $A_{3}$ should involve only cubic terms in $\hat{x}$ and $\hat{p}$, while $A_{4}$ involves only quartic terms. These terms are multiplied by any of the three Pauli matrices and the unit matrix. This leaves 12 free parameters for $A_{3}$ and 15 free parameters for $A_{4}$. By imposing that the cubic and quartic terms (apart from $x^{4}$ ) vanish, we find an explicit expression for $A_{3}$ and $A_{4}$, 


$$
\begin{aligned}
A_{3}= & \frac{4 i t \hbar^{3}}{3 \Delta_{3}^{2} \Delta^{6}}\left[4 \sigma_{x} t \hbar \omega_{\mathrm{m}}\left[\hat{x} \hat{p} \hat{x}\left(3 \hbar^{2} \omega_{\mathrm{m}}^{2}-t^{2}\right)+2 \hbar^{2} \omega_{\mathrm{m}}^{2} \hat{p}^{3}\right]\right. \\
& \left.+\sigma_{y}\left[8 t^{2} \hbar^{2} \omega_{\mathrm{m}}^{2} \hat{p} \hat{x} \hat{p}+\hat{x}^{3}\left(-t^{4}+6 t^{2} \hbar^{2} \omega_{\mathrm{m}}^{2}+3 \hbar^{4} \omega_{\mathrm{m}}^{4}\right)\right]\right],
\end{aligned}
$$

$$
A_{4}=\frac{i \sigma_{z}\left(\hat{x}^{3} \hat{p}+\hat{p} \hat{x}^{3}\right) t \hbar^{5} \omega_{\mathrm{m}}\left(11 t^{2}-3 \hbar^{2} \omega_{\mathrm{m}}^{2}\right)}{6 \Delta_{3}^{2} \Delta^{6}}
$$

This procedure leads to the Hamiltonian (5) with the coefficients given by Eqs. (6) and (7). Note that the coefficients $\alpha_{1}$ and $\alpha_{2}$ are very close to 1 in the limit $\hbar \omega_{\mathrm{m}} / t \ll 1$ since the corrections scale like $(\hbar g / t)^{4}$ and $\hbar^{4} g^{4} \omega_{\mathrm{m}}^{2} / t^{6}$.

Thus, we have shown that the Born-Oppenheimer picture gives a qualitatively correct description of the problem, even deep in the quantum regime when $\hbar \omega_{\mathrm{m}}$ is not negligible in front of $t$. This result implies a nontrivial unitary transformation that, in contrast to the BornOppenheimer picture, mixes the mechanical and charge degrees of freedom. The second important difference is that the coefficients for the quadratic and quartic terms differ from the ones of the semiclassical case. These differences are, of course, important if a quantitative description of the anharmonicity is needed.

\section{Form of the operators in qubit Hilbert space}

In order to study the decoherence and the way in which the mechanical qubit can be manipulated, it is important to obtain the projection of the main operators on the Hilbert subspace formed by the lowest two Hamiltonian eigenstates. This projection, of course, can be found numerically in a straightforward way, but it is also useful to have simple, though approximate, expressions for the form of the operators. For this purpose, one can apply the unitary transformations $U=U_{r} D$, introduced above, to find the expression of the relevant operators in the base for which the Hamiltonian reduces to the form (5) at order $g^{4}$. We are interested in the Pauli matrices for the charge sector and the $\hat{x}$ and $\hat{p}$ operators for the oscillator sector. Let us define $O^{T}=U^{\dagger} O U$.

We obtain

$$
\begin{gathered}
\sigma_{x}^{T}=\sigma_{z}+2 \hbar g \frac{\hat{p} \sigma_{y} \hbar \omega_{\mathrm{m}}-\sigma_{x} \hat{x} t}{\Delta^{2}}-2 \hbar^{2} g^{2} \frac{\sigma_{z} \hat{x}^{2} t^{2}+2 t \hbar \omega_{\mathrm{m}}+\hat{p}^{2} \sigma_{z}\left(\hbar \omega_{\mathrm{m}}\right)^{2}}{\Delta^{4}}+o\left(g^{3}\right), \\
\sigma_{y}^{T}=\sigma_{y}-2 \hbar^{2} g \omega_{\mathrm{m}} \frac{\hat{p} \sigma_{x}}{\Delta^{2}}+\hbar^{3} g^{2} \omega_{\mathrm{m}} \frac{\sigma_{x} t(\hat{x} \hat{p}+\hat{p} \hat{x})-2 \hat{p}^{2} \sigma_{y} \hbar \omega_{\mathrm{m}}}{\Delta^{4}}+o\left(g^{3}\right), \\
\sigma_{z}^{T}=-\sigma_{x}-2 \hbar g t \frac{\hat{x} \sigma_{z}}{\Delta^{2}}+\hbar^{2} g^{2} t \frac{2 \sigma_{z} \hat{x}^{2} t-\sigma_{y} \hbar \omega_{\mathrm{m}}(\hat{x} \hat{p}+\hat{p} \hat{x})}{\Delta^{4}}+o\left(g^{3}\right), \\
\hat{x}^{T}=\hat{x}+2 \hbar^{2} g \omega_{\mathrm{m}} \frac{\sigma_{x}}{\Delta^{2}}+2 \hbar^{3} g^{2} \frac{\sigma_{z} \hat{x} t \omega_{\mathrm{m}}}{\Delta^{4}}+o\left(g^{3}\right), \\
\hat{p}^{T}=\hat{p}-2 \hbar g t \frac{\sigma_{y}}{\Delta^{2}}+2 \hbar^{3} g^{2} \frac{\sigma_{z} \hat{p} t \omega_{\mathrm{m}}}{\Delta^{4}}+o\left(g^{3}\right) .
\end{gathered}
$$

The projection in the subspace of the first two excited states can be readily calculated by neglecting the quartic term of the Hamiltonian given by Eq. (5). This method implies a scaling of the $\hat{x}$ and $\hat{p}$ operators by the factor $\xi=\xi_{-}$defined by Eq. (9): $\hat{x} \rightarrow \xi \hat{x}$ and $\hat{p} \rightarrow \hat{p} / \xi$. The result at order 4 in $g$ shows that only six components are nonvanishing out of a possible 16 . These components are given by Eqs. (11) and (12) in the main text. The expression for the $\beta$ coefficients is given in the main text [Eqs. (13)-(18)] to order $g^{2}$. From these expressions, one can see how the different degrees of freedom are mixed by the interaction. For instance, the displacement acquires a $\sigma_{x}$ component, which, in this basis, is the charge operator. On the other side, the charge operator $\sigma_{z}$ acquires a component of the displacement operator. Here, we give the $g^{3}$ and $g^{4}$ terms (we use $\hbar=1$ in these expressions):

$$
\begin{aligned}
& \beta_{14}=\frac{16 t\left(6 \omega_{\mathrm{m}}^{2} t^{3}\left(9 \xi^{4}+2\right) \xi^{4}-4 \omega_{\mathrm{m}}^{3} t^{2}\left(15 \xi^{4}+16\right) \xi^{2}+9 \omega_{\mathrm{m}}^{4} t\left(3 \xi^{8}+4 \xi^{4}+8\right)-18 \omega_{\mathrm{m}}^{5} \xi^{6}+14 \omega_{\mathrm{m}} t^{4} \xi^{6}-9 t^{5} \xi^{8}\right)}{3 \Delta^{8} \Delta_{3}^{2} \xi^{4}}, \\
& \beta_{24}=\frac{16 t\left(-4 \omega_{\mathrm{m}}^{2} t^{3}\left(9 \xi^{4}+2\right) \xi^{4}+2 \omega_{\mathrm{m}}^{3} t^{2}\left(15 \xi^{4}+16\right) \xi^{2}-6 \omega_{\mathrm{m}}^{4} t\left(3 \xi^{8}+4 \xi^{4}+8\right)+9 \omega_{\mathrm{m}}^{5} \xi^{6}-7 \omega_{\mathrm{m}} t^{4} \xi^{6}+6 t^{5} \xi^{8}\right)}{3 \Delta^{8} \Delta_{3}^{2} \xi^{4}},
\end{aligned}
$$




$$
\begin{gathered}
\beta_{33}=\frac{96 \omega_{\mathrm{m}}^{3} t^{2}\left(\xi^{4}+2\right)-32 \omega_{\mathrm{m}} t^{4} \xi^{4}}{3 \Delta^{6} \Delta_{3}^{2} \xi^{3}} \\
\beta_{43}=\frac{8\left(2 \omega_{\mathrm{m}}^{2} t^{3}\left(9 \xi^{4}+4\right)+9 \omega_{\mathrm{m}}^{4} t \xi^{4}-3 t^{5} \xi^{4}\right)}{3 \Delta^{6} \Delta_{3}^{2} \xi} \\
\beta_{54}=\frac{2 \omega_{\mathrm{m}} t\left(3 \xi^{4}\left(-58 \omega_{\mathrm{m}}^{2} t^{2}-15 \omega_{\mathrm{m}}^{4}+9 t^{4}\right)-64 \omega_{\mathrm{m}}^{2} t^{2}-96 \omega_{\mathrm{m}} t \xi^{2}\left(t-\omega_{\mathrm{m}}\right)\left(\omega_{\mathrm{m}}+t\right)\right)}{3 \Delta^{8} \Delta_{3}^{2} \xi} \\
\beta_{64}=\frac{2 \omega_{\mathrm{m}} t\left(\xi^{4}\left(-66 \omega_{\mathrm{m}}^{2} t^{2}-27 \omega_{\mathrm{m}}^{4}+29 t^{4}\right)-192 \omega_{\mathrm{m}}^{2} t^{2}+96 \omega_{\mathrm{m}} t \xi^{2}\left(t-\omega_{\mathrm{m}}\right)\left(\omega_{\mathrm{m}}+t\right)\right)}{3 \Delta^{8} \Delta^{2} \xi^{3}}
\end{gathered}
$$

\section{APPENDIX C: MICROWAVE CAVITY COUPLED TO ONE AND TWO QUBITS}

Let us consider a generic system coupled linearly through the operator $S$ to a microwave cavity. The Hamiltonian can be written as

$$
H / \hbar=H_{S} / \hbar+\omega_{c} b^{\dagger} b+S\left(b^{\dagger}+b\right),
$$

where $b$ are the photon destruction operators, $\omega_{c}$ the cavity resonating angular velocity, and $H_{S}$ the unspecified system Hamiltonian. We assume that $S$ acts only in the system Hilbert space. Let us also define the energy eigenvalues of $H_{S}$ : $\hbar \epsilon_{i}$ with eigenstates $|i\rangle$ such that $H_{S}|i\rangle=\hbar \epsilon_{i}|i\rangle$.

Assuming that $S$ is small, we find the modification of the eigenvalues and eigenvectors of the full system by standard second-order perturbation theory. The unperturbed eigenvectors of the system plus cavity are $|i m\rangle$ with eigenvalue $\varepsilon_{i m}^{(0)}=\epsilon_{i}+m \omega_{c}$. The first-order correction vanishes. The second-order one reads

$$
\varepsilon_{i m}^{(2)}=\sum_{j}\left|S_{i j}\right|^{2}\left[\frac{m}{\epsilon_{i j}+\omega_{c}}+\frac{m+1}{\epsilon_{i j}-\omega_{c}}\right],
$$

with $\epsilon_{i j}=\epsilon_{i}-\epsilon_{j}$. The linear part in $m$ of this expression gives the renormalization of the resonator frequency. It normally depends on the system state $i$ :

$$
\Delta \omega_{i}=\sum_{j}\left|S_{i j}\right|^{2} \frac{2 \epsilon_{i j}}{\left(\epsilon_{i j}^{2}-\omega_{c}^{2}\right)} .
$$

Thus, the dispersive coupling $\chi$ [cf. Eq. (21)], defined as half the variation of the resonating frequency for a transition from the ground to the first excited state of the system, is

$$
\chi=\left(\Delta \omega_{1}-\Delta \omega_{0}\right) / 2 .
$$

\section{Dispersive coupling for a single qubit}

As a simple example, one can consider the case $H_{S} / \hbar=$ $\epsilon_{10} \tau_{z} / 2$ and $S=g_{\mathrm{v}} \tau_{x}$. One finds $\Delta \omega_{1}=-\Delta \omega_{0}=2 g_{\mathrm{v}}^{2} \epsilon_{10} /$ $\left(\epsilon_{10}^{2}-\omega_{c}^{2}\right)$. For $\omega_{c}$ close to $\epsilon_{10}$, one then recovers the value of $\chi=g_{\mathrm{v}}^{2} /\left(\epsilon_{10}-\omega_{c}^{2}\right)$ entering Eq. (21).

Using Eq. (C3), we can now find the dispersive coupling for the nanomechanical qubit. We perform the unitary transformation given by $D_{1} U_{r}$, and we use for $H_{S}$ the quadratic Hamiltonian $\mathrm{H}_{2}$ given in Eq. (B2). In this case, the eigenvectors are $|n \sigma\rangle$ with eigenvalues $E_{n \sigma}=\hbar n \omega_{\sigma}+$ $t_{R} \sigma / 2$ [here, $t_{R}=t+\hbar\left(\omega_{+}-\omega_{-}\right)$is the hopping amplitude renormalized by the zero-point energies]. The system couples to the cavity through the charge and the displacement operators, but since the latter coupling is much smaller than the former, we consider, in the following, only the charge operator $\sigma_{z}$. We write the coupling operator in the new basis: $S=g_{\mathrm{ec}} D_{1}^{\dagger} U_{r}^{\dagger} \sigma_{z} U_{r} D_{1}$. At lowest order, it reads [cf. Eq. (B12)]

$$
\frac{S}{g_{\mathrm{ec}}}=\sigma_{x}+\frac{2 \hbar g t}{\Delta^{2}}\left(\begin{array}{cc}
\left(a_{+}+a_{+}\right) \xi_{+} & 0 \\
0 & \left(a_{-}+a_{-}\right) \xi_{-}
\end{array}\right)+\ldots
$$

Substituting $S$ into Eqs. (C3) and (C4) with the two lowestlying states $|0-\rangle$ and $|1-\rangle$, we find $\chi=\chi_{m}+\chi_{c}$ with

$$
\chi_{m}=2 g_{\mathrm{ec}}^{2} \beta_{4,1}^{2} \frac{\left(\omega_{21}-\omega_{10}\right)\left(\omega_{c}^{2}+\omega_{10} \omega_{21}\right)}{\left(\omega_{c}^{2}-\omega_{21}^{2}\right)\left(\omega_{c}^{2}-\omega_{10}^{2}\right)}
$$

and

$$
\chi_{c}=g_{e c}^{2} \frac{\left(\delta_{11}-\delta_{00}\right)\left(\omega_{c}^{2}+\delta_{11} \delta_{00}\right)}{\left(\omega_{c}^{2}-\delta_{11}^{2}\right)\left(\omega_{c}^{2}-\delta_{00}^{2}\right)}
$$

where we recall that $\beta_{4,1}=2 \hbar g t \xi_{-} / \Delta^{2}$ and $\delta_{n m}=$ $\left(E_{n+}-E_{n-}\right) / \hbar$. Note that the expression in Eq. (C6) vanishes if the lowest-order approximation for the energy 
eigenvalues is used. A nonlinearity is needed in order to have a finite dispersive coupling. For this reason, we do not specify the values of $\omega_{n m}$ and $\delta_{n m}$ for the moment. Both expressions have a divergent behavior: $\chi_{m}$ for $\omega_{c}$ close to either $\omega_{01}$ or $\omega_{21}$, and $\chi_{e}$ for $\omega_{c}$ close to either $\delta_{00}$ or $\delta_{11}$. This behavior allows us to write the approximate Eqs. (22) and (23) in the main text.

\section{Coupling two qubits via the cavity}

We now apply this approach to study two nanomechanical qubits coupled to the same microwave cavity. Our main goal is to find the expression of a system operator $F$, acting only in the system Hilbert space, on the eigenvector basis of the coupled system of the two qubits plus the microwave cavity. We look at the $m$-independent part, which gives the change of the operator in the system subspace. Applying second-order perturbation theory with the same notation as before, we obtain

$$
\begin{aligned}
\left\langle i^{\prime} m|F| i m\right\rangle \\
=F_{i^{\prime} i}+\sum_{k, l \neq i^{\prime}} \frac{S_{i^{\prime} k} S_{k l} F_{l i}}{\left(\epsilon_{i^{\prime} k}-\omega_{c}\right) \epsilon_{i^{\prime} l}} \\
\quad+\sum_{k, l \neq i} \frac{F_{i^{\prime} l} S_{l k} S_{k i}}{\left(\epsilon_{i k}-\omega_{c}\right) \epsilon_{i l}}+\sum_{k k^{\prime}} \frac{S_{i^{\prime} k} F_{k k^{\prime}} S_{k^{\prime} i}}{\left(\epsilon_{i^{\prime} k}-\omega_{c}\right)\left(\epsilon_{i k^{\prime}}-\omega_{c}\right)} \\
\quad-\frac{F_{i^{\prime} i}}{2}\left[\sum_{j \neq i} \frac{\left|S_{i j}\right|^{2}}{\left(\epsilon_{i j}-\omega_{c}\right)^{2}}+\sum_{j \neq i^{\prime}} \frac{\left|S_{i^{\prime} j}\right|^{2}}{\left(\epsilon_{i^{\prime} j}-\omega_{c}\right)^{2}}\right] .
\end{aligned}
$$

As a simple application, we can consider a system composed of two, pure, two-level system qubits: $H_{S}=$ $\sum_{a=1,2} \hbar \epsilon_{10}^{(a)} \tau_{z}^{(a)} / 2$, with $S=\sum_{a=1,2} g_{\mathrm{v}}^{(a)} \tau_{x}^{(a)}$. When a drive is applied to qubit 1 , this can be modeled by a term in the Hamiltonian $\hbar A \cos \left(\omega_{D} t\right) \tau_{x}^{(1)}$. We thus look at how $F=\tau_{x}$ reads in the Hamiltonian eigenvector basis. Using Eq. (C8), we find that

$$
F=F_{x 0} \tau_{x}^{(1)}+F_{0 x} \tau_{x}^{(2)}+F_{z x} \tau_{x}^{(1)} \tau_{x}^{(2)},
$$

with $F_{z x}$ given by the expression (42) for $J_{z x}$ with $\omega_{10} \rightarrow$ $\epsilon_{10}$ and $A \rightarrow 1$.

We now consider the case of a nanomechanical qubit. To evaluate Eq. (C8), we use the same method applied for the single qubit. The coupling operator is now $S=$ $\sum_{a=1,2} g_{\mathrm{ec}}^{(a)} \sigma_{z}^{(a)}$. The eigenstates of the composite system can be labeled with the four indices $\left\{n_{1}, \sigma_{1} ; n_{2} \sigma_{2}\right\}$ with eigenvalues $E_{n_{1}, \sigma_{1}}+E_{n_{2}, \sigma_{2}}$. As before, we assume we have the exact expressions for the eigenvalues, and we use the matrix elements given by the quadratic Hamiltonian. We look for the contributions leading to the operator $\tau_{z}^{(1)} \tau_{x}^{(2)}$. We find that, also in this case, $F$ has the form of Eq. (C9).

At lowest order in the electromechanical coupling constants, these terms are generated by selecting the contribution of two $\sigma_{x}^{(1)}$ and one $\hat{x}^{(2)}$ operators entering the matrix elements of $F$ and $S$. They have dominant divergent terms in $1 /\left(\omega_{c}-\omega_{10}^{(2)}\right)$. Collecting them, one obtains

$F_{z x}^{e}=\frac{g_{\mathrm{ec}}^{(1)} g_{\mathrm{ec}}^{(2)} \beta_{4,1}^{(2)}}{\omega_{10}^{(2)}-\omega_{c}}\left[\frac{\delta_{11}^{(1)}}{\delta_{11}^{(1) 2}-\omega_{10}^{(2) 2}}-\frac{\delta_{00}^{(1)}}{\delta_{11}^{(1) 2}-\omega_{10}^{(2) 2}}\right]$,

which, close to the resonance, can be written as

$$
F_{z x}^{e} \approx-\frac{g_{\mathrm{ec}}^{(1)} g_{\mathrm{ec}}^{(2)} \beta_{1,4}^{(2)}}{\omega_{10}^{(2)}-\omega_{c}} \frac{\left(\delta_{11}^{(1)}-\delta_{00}^{(1)}\right)\left(\delta_{00}^{(1) 2}+\omega_{10}^{(2) 2}\right)}{\left(\delta_{00}^{(1) 2}-\omega_{10}^{(2) 2}\right)^{2}} .
$$

Even if this term appears to be a first-order contribution in $g^{(2)}$, we know that the numerator is of order $g^{(1) 2}$ [cf. Eq. (24)]. Thus, we also need to evaluate the nextorder contributions in Eq. (C8) that imply, for the operators $F$ and $S$, two $\hat{x}^{(1)}$ and one $\hat{x}^{(2)}$ operators. These terms are of order $g^{(2)} g^{(1) 2}$. Collecting the divergent contribution as before and evaluating it close to the divergence, we have

$F_{z x}^{m}=\frac{8 g_{\mathrm{ec}}^{(1)} g_{\mathrm{ec}}^{(2)}}{\omega_{c}-\omega_{10}^{(2)}} \beta_{1,4}^{(1) 2} \beta_{1,4}^{(2)} \frac{\omega_{10}^{(1)}-\omega_{21}^{(1)}}{\left(\omega_{10}^{(2)}-\omega_{21}^{(1)}\right)\left(\omega_{10}^{(1)}-\omega_{10}^{(2)}\right)}$.

The two terms $F_{z x}^{m}$ and $F_{z x}^{e}$ can be combined in the form given by Eq. (43) in the main text and written using the results obtained for the dispersive shifts $\chi_{m}$ and $\chi_{e}$ as defined in Eqs. (C6) and (C7).

[1] S. Barzanjeh, D. Vitali, P. Tombesi, and G. J. Milburn, Entangling Optical and Microwave Cavity Modes by Means of a Nanomechanical Resonator, Phys. Rev. A 84, 042342 (2011).

[2] T. A. Palomaki, J. W. Harlow, J. D. Teufel, R. W. Simmonds, and K. W. Lehnert, Coherent State Transfer between Itinerant Microwave Fields and a Mechanical Oscillator, Nature (London) 495, 210 (2013).

[3] R. W. Andrews, R. W. Peterson, T. P. Purdy, K. Cicak, R. W. Simmonds, C. A. Regal, and K. W. Lehnert, Bidirectional and Efficient Conversion between Microwave and Optical Light, Nat. Phys. 10, 321 (2014).

[4] F. Lecocq, J. B. Clark, R. W. Simmonds, J. Aumentado, and J. D. Teufel, Mechanically Mediated Microwave Frequency Conversion in the Quantum Regime, Phys. Rev. Lett. 116, 043601 (2016).

[5] A. Vainsencher, K. J. Satzinger, G. A. Peairs, and A. N. Cleland, Bi-Directional Conversion between Microwave and Optical Frequencies in a Piezoelectric Optomechanical Device, Appl. Phys. Lett. 109, 033107 (2016). 
[6] J. Bochmann, A. Vainsencher, D. D. Awschalom, and A. N. Cleland, Nanomechanical Coupling between Microwave and Optical Photons, Nat. Phys. 9, 712 (2013).

[7] C. F. Ockeloen-Korppi, E. Damskägg, J.-M. Pirkkalainen, A. A. Clerk, M. J. Woolley, and M. A. Sillanpää, Quantum Backaction Evading Measurement of Collective Mechanical Modes, Phys. Rev. Lett. 117, 140401 (2016).

[8] P. Rabl, S. J. Kolkowitz, F. H. L. Koppens, J. G. E. Harris, P. Zoller, and M. D. Lukin, A Quantum Spin Transducer Based on Nanoelectromechanical Resonator Arrays, Nat. Phys. 6, 602 (2010).

[9] K. Stannigel, P. Rabl, A. S. Sørensen, P. Zoller, and M. D. Lukin, Optomechanical Transducers for Long-Distance Quantum Communication, Phys. Rev. Lett. 105, 220501 (2010).

[10] K. J. Satzinger, Y. P. Zhong, H.-S. Chang, G. A. Peairs, A. Bienfait, M.-H. Chou, A. Y. Cleland, C. R. Conner, É. Dumur, J. Grebel, I. Gutierrez, B. H. November, R. G. Povey, S. J. Whiteley, D. D. Awschalom, D. I. Schuster, and A. N. Cleland, Quantum Control of Surface AcousticWave Phonons, Nature (London) 563, 661 (2018).

[11] A. Bienfait, K. J. Satzinger, Y. P. Zhong, H.-S. Chang, M.-H. Chou, C. R. Conner, É. Dumur, J. Grebel, G. A. Peairs, R. G. Povey, and A. N. Cleland, Phonon-Mediated Quantum State Transfer and Remote Qubit Entanglement, Science 364, 368 (2019).

[12] A. Bienfait, Y. P. Zhong, H.-S. Chang, M.-H. Chou, C. R. Conner, É. Dumur, J. Grebel, G. A. Peairs, R. G. Povey, K. J. Satzinger, and A. N. Cleland, Quantum Erasure Using Entangled Surface Acoustic Phonons, Phys. Rev. X 10, 021055 (2020).

[13] C. L. Degen, F. Reinhard, and P. Cappellaro, Quantum Sensing, Rev. Mod. Phys. 89, 035002 (2017).

[14] C. Urgell, W. Yang, S. L. De Bonis, C. Samanta, M. J. Esplandiu, Q. Dong, Y. Jin, and A. Bachtold, Cooling and Self-Oscillation in a Nanotube Electromechanical Resonator, Nat. Phys. 16, 32 (2020).

[15] G. S. MacCabe, H. Ren, J. Luo, J. D. Cohen, H. Zhou, A. Sipahigil, M. Mirhosseini, and O. Painter, Nano-Acoustic Resonator with Ultralong Phonon Lifetime, Science 370 , 840 (2020).

[16] F. Arute et al., Quantum Supremacy Using a Programmable Superconducting Processor, Nature (London) 574, 505 (2019).

[17] C. Rigetti, J. M. Gambetta, S. Poletto, B. L. T. Plourde, J. M. Chow, A. D. Córcoles, J. A. Smolin, S. T. Merkel, J. R. Rozen, G. A. Keefe, M. B. Rothwell, M. B. Ketchen, and M. Steffen, Superconducting Qubit in a Waveguide Cavity with a Coherence Time Approaching 0.1 ms, Phys. Rev. B 86, 100506(R) (2012).

[18] S. Rips and M. J. Hartmann, Quantum Information Processing with Nanomechanical Qubits, Phys. Rev. Lett. 110, 120503 (2013).

[19] S. Rips, I. Wilson-Rae, and M. J. Hartmann, Nonlinear Nanomechanical Resonators for Quantum Optoelectromechanics, Phys. Rev. A 89, 013854 (2014).

[20] A. D. Armour, M. P. Blencowe, and Y. Zhang, Classical Dynamics of a Nanomechanical Resonator Coupled to a Single-Electron Transistor, Phys. Rev. B 69, 125313 (2004).
[21] Ya. M. Blanter, O. Usmani, and Yu. V. Nazarov, SingleElectron Tunneling with Strong Mechanical Feedback, Phys. Rev. Lett. 93, 136802 (2004); 94, 049904(E) (2005).

[22] N. M. Chtchelkatchev, W. Belzig, and C. Bruder, Charge Transport through a Single-Electron Transistor with a Mechanically Oscillating Island, Phys. Rev. B 70, 193305 (2004).

[23] A. A. Clerk and S. Bennett, Quantum Nanoelectromechanics with Electrons, Quasi-particles and Cooper Pairs: Effective Bath Descriptions and Strong Feedback Effects, New J. Phys. 7, 238 (2005).

[24] J. Koch and F. von Oppen, Franck-Condon Blockade and Giant Fano Factors in Transport through Single Molecules, Phys. Rev. Lett. 94, 206804 (2005).

[25] D. Mozyrsky, M. B. Hastings, and I. Martin, Intermittent Polaron Dynamics: Born-Oppenheimer Approximation out of Equilibrium, Phys. Rev. B 73, 035104 (2006).

[26] C. B. Doiron, W. Belzig, and C. Bruder, Electrical Transport through a Single-Electron Transistor Strongly Coupled to an Oscillator, Phys. Rev. B 74, 205336 (2006).

[27] F. Pistolesi and S. Labarthe, Current Blockade in Classical Single-Electron Nanomechanical Resonator, Phys. Rev. B 76, 165317 (2007).

[28] S. L. de Bonis, C. Urgell, W. Yang, C. Samanta, A. Noury, J. Vergara-Cruz, Q. Dong, Y. Jin, and A. Bachtold, Ultrasensitive Displacement Noise Measurement of Carbon Nanotube Mechanical Resonators, Nano Lett. 18, 5324 (2018).

[29] I. Khivrich, A. A. Clerk, and S. Ilani, Nanomechanical Pump-Probe Measurements of Insulating Electronic States in a Carbon Nanotube, Nat. Nanotechnol. 14, 161 (2019).

[30] S. Blien, P. Steger, N. Hüttner, R. Graaf, and A. K. Hüttel, Quantum Capacitance Mediated Carbon Nanotube Optomechanics, Nat. Commun. 11, 1636 (2020).

[31] Y. Wen, N. Ares, F. J. Schupp, T. Pei, G. A. D. Briggs, and E. A. Laird, A Coherent Nanomechanical Oscillator Driven by Single-Electron Tunnelling, Nat. Phys. 16, 75 (2020).

[32] A. Benyamini, A. Hamo, S. V. Kusminskiy, F. von Oppen, and S. Ilani, Real-Space Tailoring of the Electron-Phonon Coupling in Ultraclean Nanotube Mechanical Resonators, Nat. Phys. 10, 151 (2014).

[33] A. Hamo, A. Benyamini, I. Shapir, I. Khivrich, J. Waissman, K. Kaasbjerg, Y. Oreg, F. von Oppen, and S. Ilani, Electron Attraction Mediated by Coulomb Repulsion, Nature (London) 535, 395 (2016).

[34] W. G. van der Wiel, S. De Franceschi, J. M. Elzerman, T. Fujisawa, S. Tarucha, and L. P. Kouwenhoven, Electron Transport through Double Quantum Dots, Rev. Mod. Phys. 75, 1 (2002).

[35] M. Galperin, M. A. Ratner, and A. Nitzan, Hysteresis, Switching, and Negative Differential Resistance in Molecular Junctions: A Polaron Model, Nano Lett. 5, 125 (2005).

[36] G. Micchi, R. Avriller, and F. Pistolesi, Mechanical Signatures of the Current Blockade Instability in Suspended Carbon Nanotubes, Phys. Rev. Lett. 115, 206802 (2015).

[37] R. Avriller, B. Murr, and F. Pistolesi, Bistability and Displacement Fluctuations in a Quantum Nanomechanical Oscillator, Phys. Rev. B 97, 155414 (2018).

[38] F. T. Hioe and E. W. Montroll, Quantum Theory of Anharmonic Oscillators. I. Energy Levels of Oscillators with 
Positive Quartic Anharmonicity, J. Math. Phys. (N.Y.) 16, 1945 (1975).

[39] J. A. Schreier, A. A. Houck, J. Koch, D. I. Schuster, B. R. Johnson, J. M. Chow, J. M. Gambetta, J. Majer, L. Frunzio, M. H. Devoret, S. M. Girvin, and R. J. Schoelkopf, Suppressing Charge Noise Decoherence in Superconducting Charge Qubits, Phys. Rev. B 77, 180502(R) (2008).

[40] F. T. Hioe, D. MacMillen, and E. W. Montroll, Quantum Theory of Anharmonic Oscillators: Energy Levels of a Single and a Pair of Coupled Oscillators with Quartic Coupling, Phys. Rep. 43, 305 (1978).

[41] E. Collin, G. Ithier, A. Aassime, P. Joyez, D. Vion, and D. Esteve, NMR-like Control of a Quantum Bit Superconducting Circuit, Phys. Rev. Lett. 93, 157005 (2004).

[42] J. Majer, J. M. Chow, J. M. Gambetta, J. Koch, B. R. Johnson, J. A. Schreier, L. Frunzio, D. I. Schuster, A. A. Houck, A. Wallraff, A. Blais, M. H. Devoret, S. M. Girvin, and R. J. Schoelkopf, Coupling Superconducting Qubits via a Cavity Bus, Nature (London) 449, 443 (2007).

[43] A. A. Houck, J. A. Schreier, B. R. Johnson, J. M. Chow, J. Koch, J. M. Gambetta, D. I. Schuster, L. Frunzio, M. H. Devoret, S. M. Girvin, and R. J. Schoelkopf, Controlling the Spontaneous Emission of a Superconducting Transmon Qubit, Phys. Rev. Lett. 101, 080502 (2008).

[44] A. Blais, R.-S. Huang, A. Wallraff, S. M. Girvin, and R. J. Schoelkopf, Cavity Quantum Electrodynamics for Superconducting Electrical Circuits: An Architecture for Quantum Computation, Phys. Rev. A 69, 062320 (2004).

[45] J. Koch, T. M. Yu, J. Gambetta, A. A. Houck, D. I. Schuster, J. Majer, A. Blais, M. H. Devoret, S. M. Girvin, and R. J. Schoelkopf, Charge-Insensitive Qubit Design Derived from the Cooper Pair Box, Phys. Rev. A 76, 042319 (2007).

[46] P. Scarlino, D. J. van Woerkom, A. Stockklauser, J. V. Koski, M. C. Collodo, S. Gasparinetti, C. Reichl, W. Wegscheider, T. Ihn, K. Ensslin, and A. Wallraff, All-Microwave Control and Dispersive Readout of GateDefined Quantum Dot Qubits in Circuit Quantum Electrodynamics, Phys. Rev. Lett. 122, 206802 (2019).

[47] J. Hauss, A. Fedorov, S. André, V. Brosco, C. Hutter, R. Kothari, S. Yeshwanth, A. Shnirman, and G. Schön, Dissipation in Circuit Quantum Electrodynamics: Lasing and Cooling of a Low-Frequency Oscillator, New J. Phys. 10, 095018 (2008).

[48] C. Cohen-Tannoudji, J. Dupont-Roc, and G. Grynberg, Atom-Photon Interactions: Basic Processes and Applications (Wiley, New York, 1992).
[49] C. Rigetti and M. Devoret, Fully Microwave-Tunable Universal Gates in Superconducting Qubits with Linear Couplings and Fixed Transition Frequencies, Phys. Rev. B 81, 134507 (2010).

[50] J. M. Chow, A. D. Córcoles, J. M. Gambetta, C. Rigetti, B. R. Johnson, J. A. Smolin, J. R. Rozen, G. A. Keefe, M. B. Rothwell, M. B. Ketchen, and M. Steffen, Simple All-Microwave Entangling Gate for Fixed-Frequency Superconducting Qubits, Phys. Rev. Lett. 107, 080502 (2011).

[51] W. Yang, C. Urgell, S. L. De Bonis, M. Marganska, M. Grifoni, and A. Bachtold, Fabry-Pérot Oscillations in Correlated Carbon Nanotubes, Phys. Rev. Lett. 125, 187701 (2020).

[52] J. J. Viennot, M. C. Dartiailh, A. Cottet, and T. Kontos, Coherent Coupling of a Single Spin to Microwave Cavity Photons, Science 349, 408 (2015).

[53] T. Cubaynes, M. R. Delbecq, M. C. Dartiailh, R. Assouly, M. M. Desjardins, L. C. Contamin, L. E. Bruhat, Z. Leghtas, F. Mallet, A. Cottet, and T. Kontos, Highly Coherent Spin States in Carbon Nanotubes Coupled to Cavity Photons, npj Quantum Inf. 5, 47 (2019).

[54] E. Hebestreit, M. Frimmer, R. Reimann, and L. Novotny, Sensing Static Forces with Free-Falling Nanoparticles, Phys. Rev. Lett. 121, 063602 (2018).

[55] H. J. Hug, B. Stiefel, P. J. A. van Schendel, A. Moser, S. Martin, and H.-J. Güntherodt, A Low Temperature Ultrahigh Vacuum Scanning Force Microscope, Rev. Sci. Instrum. 70, 3625 (1999).

[56] M. Ribezzi-Crivellari, J. M. Huguet, and F. Ritort, CounterPropagating Dual-Trap Optical Tweezers Based on Linear Momentum Conservation, Rev. Sci. Instrum. 84, 043104 (2013).

[57] A. A. Clerk, M. H. Devoret, S. M. Girvin, F. Marquardt, and R. J. Schoelkopf, Introduction to Quantum Noise, Measurement, and Amplification, Rev. Mod. Phys. 82, 1155 (2010).

[58] H. Grabert and M. H. Devoret, Single Charge Tunneling: Coulomb Blockade Phenomena in Nanostructures (Springer Science \& Business Media, New York, 2013).

[59] D. Braak, Integrability of the Rabi Model, Phys. Rev. Lett. 107, 100401 (2011).

[60] D. Zueco, G. M. Reuther, S. Kohler, and P. Hänggi, QubitOscillator Dynamics in the Dispersive Regime: Analytical Theory beyond the Rotating-Wave Approximation, Phys. Rev. A 80, 033846 (2009). 